\title{
Mesothelial cells promote early ovarian cancer metastasis through fibronectin secretion
}

\author{
Hilary A. Kenny, ${ }^{1}$ Chun-Yi Chiang, ${ }^{1}$ Erin A. White, ${ }^{1}$ Elizabeth M. Schryver, ${ }^{1}$ Mohammed Habis, ${ }^{1}$ Iris L. Romero, ${ }^{1}$ Andras Ladanyi, ${ }^{1}$ \\ Carla V. Penicka, ${ }^{1}$ Joshy George, ${ }^{2,3}$ Karl Matlin, ${ }^{4}$ Anthony Montag, ${ }^{5}$ Kristen Wroblewski, ${ }^{6}$ S. Diane Yamada, ${ }^{1}$ Andrew P. Mazar, \\ David Bowtell, ${ }^{2,3}$ and Ernst Lengyel ${ }^{1}$
}

\begin{abstract}
'Department of Obstetrics and Gynecology, Section of Gynecologic Oncology, University of Chicago, Chicago, Illinois, USA. ${ }^{2}$ Peter MacCallum Cancer Centre, East Melbourne, Australia. ${ }^{3}$ Department of Pathology and Biochemistry and Department of Molecular Biology, University of Melbourne, Melbourne, Australia. ${ }^{4}$ Department of Surgery, ${ }^{5}$ Department of Pathology, and ${ }^{6}$ Department of Health Studies, University of Chicago, Chicago, Illinois, USA. ${ }^{7}$ Chemistry of Life Processes Institute, Northwestern University, Evanston, Illinois, USA.
\end{abstract}

\begin{abstract}
Ovarian cancer ( $\mathrm{vvCa}$ ) metastasizes to organs in the abdominal cavity, such as the omentum, which are covered by a single layer of mesothelial cells. Mesothelial cells are generally thought to be "bystanders" to the metastatic process and simply displaced by OvCa cells to access the submesothelial extracellular matrix. Here, using organotypic 3D cultures, we found that primary human mesothelial cells secrete fibronectin in the presence of OvCa cells. Moreover, we evaluated the tumor stroma of 108 human omental metastases and determined that fibronectin was consistently overexpressed in these patients. Blocking fibronectin production in primary mesothelial cells in vitro or in murine models, either genetically (fibronectin 1 floxed mouse model) or via siRNA, decreased adhesion, invasion, proliferation, and metastasis of OvCa cells. Using a coculture model, we determined that OvCa cells secrete TCF- $\beta 1$, which in turn activates a TCF- $\beta$ receptor/RAC1/ SMAD-dependent signaling pathway in the mesothelial cells that promotes a mesenchymal phenotype and transcriptional upregulation of fibronectin. Additionally, blocking $\alpha_{5}$ or $\beta_{1}$ integrin function with antibodies reduced metastasis in an orthotopic preclinical model of OvCa metastasis. These findings indicate that cancer-associated mesothelial cells promote colonization during the initial steps of OvCa metastasis and suggest that mesothelial cells actively contribute to metastasis.
\end{abstract}

\section{Introduction}

The biology of serous high-grade ovarian cancer (OvCa) is different from that of most other solid tumors, since OvCa is predominantly confined within the abdominal and pleural cavities and rarely metastasizes hematogenously (1). Moreover, OvCa is generally only superficially invasive, although advanced disease is characterized by large intra-abdominal tumors in the ovary and the omentum. During OvCa dissemination, the cancer cells detach from the primary site, which can be the fallopian tube, the ovary, or the peritoneum. Subsequently, the peritoneal fluid carries the OvCa cells to secondary sites of implantation, including the omentum, the most common site of OvCa metastasis. These sites are exclusively organs with a single layer of mesothelial cells covering an underlying stroma composed of extracellular matrices (ECM) and stromal cells $(2,3)$. Consequently, OvCa cells must invade through the barrier of mesothelial cells on the peritoneum, omentum, and bowel serosa to effectively form metastases.

Mesothelial cells were originally depicted as a mechanical barrier that must be pushed to the side by tumor cells $(4,5)$. In coculture, cancer cells induced human mesothelial cells to retract from the peritoneum and omentum, thereby exposing the underlying ECM (4). Iwanicki and colleagues extended these findings by

Conflict of interest: The authors have declared that no conflict of interest exists Submitted: December 16, 2013; Accepted: July 31, 2014.

Reference information: J Clin Invest. 2014;124(10):4614-4628. doi:10.1172/JCI74778. showing that OvCa spheroids use myosin-generated force to clear mesothelial cells in human mesothelial cell line monolayers $(5,6)$. Tumor-induced apoptosis may also be important for mesothelial cell clearance and peritoneal invasion (7).

However, reports that mesothelial cells may induce the motility of OvCa cells supports a possible tumor-promoting role for these cells during OvCa metastasis. Rieppi et al. revealed that conditioned media $(\mathrm{CM})$ of primary human mesothelial cells induced migration of OvCa cell lines through a gelatin-coated Boyden chamber (8), and a later paper demonstrated that mesothelial cells promote OvCa adhesion (9). Collectively, these findings were the first evidence that mesothelial cells actively participate in the establishment of the OvCa metastatic niche. This concept is consistent with the observation that cancer cells recruit local stromal cells to promote and stabilize their growth (10). The interaction between cancer and stromal cells has primarily been studied in cancerassociated fibroblasts (CAFs), which have been shown to promote almost every aspect of local tumor growth (11). In the OvCa microenvironment, CAFs $(12,13)$ and cancer-associated adipocytes (14, 15) promote invasion and metastasis, which indicates that $\mathrm{OvCa}$ cells have the capability to recruit various types of stromal cells. It is therefore unlikely that mesothelial cells are simply "bystanders" that must be pushed out of the way by invading OvCa cells in the metastatic process. Rather, it is likely that they are recruited by OvCa cells and reprogrammed to facilitate tumor growth. Indeed, cancer cell CM may stimulate mesothelial cell motility $(16,17)$. 
Increased expression of fibronectin (encoded by FN1), a key component of the ECM, has been detected in OvCa metastases compared with the primary ovarian tumor $(14,18)$, and fibronectin is also present in ascites (19). While multiple integrins bind fibronectin at specific amino acid sequences (i.e., PHSRN and RGD), $\alpha_{5} \beta_{1}$ integrin is selective for fibronectin (20). The binding of integrins to the ECM promotes integrin clustering and subsequently triggers integrin-mediated intracellular signal transduction (21), while at the same time, integrin receptors mediate fibronectin matrix assembly (22). Functionally, fibronectin induces adhesion, proliferation, and invasion of tumor cells and contributes to the formation, adhesion, and disaggregation of OvCa spheroids $(16,23,24)$.

Given our previous finding that fibronectin is overexpressed in OvCa metastases (14), as well as indications that mesothelial cells might communicate with OvCa cells, we hypothesized that OvCa-induced fibronectin expression by mesothelial cells may play a critical role in the early steps of OvCa metastasis. We report here that OvCa cells stimulated mesothelial cells to produce fibronectin through secretion of TGF- $\beta 1$, thereby activating a TGF- $\beta$ receptor 1 (TGF- $\beta$ RI)/RAC1/SMAD-dependent signaling pathway in the mesothelial cells. The activated mesothelial cells promoted metastasis by supporting tumor cell adhesion, invasion, and proliferation.

\section{Results}

OvCa cells induce fibronectin production early in metastasis to the human omentum. The most common site of OvCa metastasis in patients with serous high-grade cancers is the omentum. In fact, in mouse models, OvCa cells home to the omentum immediately after i.p. injection (14). In a previous study, we used a reverse phase protein array to identify protein expression in paired ovarian and omental metastases and found that fibronectin expression was higher in the omental metastasis than in the primary tumor, while other ECM proteins, such as vitronectin, were more highly expressed in the primary tumor (14). This was confirmed by immunoblot using snap-frozen tissue of the paired samples from the same cohort of patients (Supplemental Figure 1A and Supplemental Methods; supplemental material available online with this article; doi:10.1172/JCI74778DS1). The results were further validated using a tissue microarray (TMA) constructed with tissue from omental metastases from 108 patients with advanced OvCa. In $93 \%$ of these patients (100 of 108), fibronectin expression was much stronger in the stromal than in the epithelial cell compartment (Figure 1A). To determine whether fibronectin expression is an early or late event during omental/peritoneal metastasis, we identified samples from a small subgroup of women diagnosed with stage IIIA serous-papillary OvCa (FIGO 1988 staging), defined by microscopic disease to the omentum (Supplemental Figure 1B), which allowed us to study early OvCa metastasis. Immunohistochemical staining showed that fibronectin expression was induced below proliferating metastatic cells that had not yet invaded the basement membrane (Figure 1B). Once the tumor invaded, the stroma around the serous tumor islands had very strong fibronectin expression (Figure 1C), confirming the TMA results. Direct comparison of protein and RNA levels of fibronectin in normal omental and omental metastatic tissues showed an increased level of fibronectin in the omental tissue harboring OvCa metastases (Figure 1C and Supplemental Figure 1C). In addition, fibronectin was abundant in the ascites of OvCa patients (Supplemental Figure 1D and ref. 19).

To determine whether the fibronectin expression in metastatic OvCa is of functional significance, ECM was extracted from unfixed benign and tumor transformed omental tissue removed at surgery from separate patients. In the ECM from omental metastasis, fibronectin expression was found to be higher, and multiple cleavage forms of fibronectin were detected (Figure 1D). Compared with ECM from normal omentum, 2 OvCa cell lines, HeyA8 and SKOV3ip1, showed increased adhesion and proliferation to the omental ECM extracted from metastatic tumors (Figure $1 \mathrm{E}$ and Supplemental Figure 1E). Cells bind to fibronectin through specific cell surface integrins, including the fibronectin receptor $\alpha_{5} \beta_{1}$ integrin and the vitronectin receptor $\alpha_{v} \beta_{3}$ integrin (21). Adhesion and proliferation of OvCa cells to tumor-derived ECM could be abrogated using blocking antibodies against these integrins as well as an RGD peptide, whereas control IgG, the control RAD peptide, and a $\beta_{4}$ integrin antibody had no inhibitory effect (Figure $1 \mathrm{E}$ and Supplemental Figure 1E).

Next, we investigated whether early metastasis of OvCa cells to the omentum could be modeled ex vivo with human omental tissue and in vivo in mice. Injection of HeyA8 OvCa cells i.p. induced strong expression of fibronectin in the mouse omentum (Figure 2A). Attachment of fluorescently labeled OvCa cells to a piece of human omentum followed by fluorescence-activated cell sorting (FACS) to separate OvCa and omental cells also showed strong protein and RNA induction of fibronectin in the surface cells that interacted with the cancer cells, whereas expression in the cancer cells was unchanged (Figure 2B).

The mesothelium covers all organs within the abdominal cavity, including the peritoneum and the omentum. It is composed of a monolayer of mesothelial cells, on top of an ECM interspersed with resting fibroblasts that are responsible for secreting most of the ECM in the basement membrane (Figure 1B, Supplemental Figure 1B, and refs. 25, 26). We and others have modeled the mesothelium in a $3 \mathrm{D}$ culture $(5,25)$ using primary human omental fibroblasts and mesothelial cells (Supplemental Figure 2). Coculture of SKOV3ip1 cells with the organotypic 3D culture induced fibronectin synthesis and matrix assembly (Figure 2C). Fibronectin matrix secretion and assembly can be assessed by detecting the conversion of cell-associated, deoxycholic acid (DOC)-soluble fibronectin fibrils into a DOC-insoluble fibril network (22). Coculture of OvCa cells with the 3D culture for 48 hours induced the secretion of soluble fibronectin in the ECM of the 3D culture and also induced the aggregation of fibronectin as a dense DOCinsoluble matrix (Figure 2C). Moreover, binding of inactive fibronectin dimers to integrins on the OvCa cell surface induced a dense fibronectin matrix (Figure 2D). These data suggest that binding of OvCa cells to the omentum induces early fibronectin production and matrix assembly, which is functionally important for adhesion, migration, and invasion.

OvCa cells stimulate fibronectin expression in mesothelial cells. Since mesothelial cells are the first cell type to interact with metastasizing OvCa cells (27), we sought to determine how the interaction between OvCa cells and mesothelial cells results in 
A

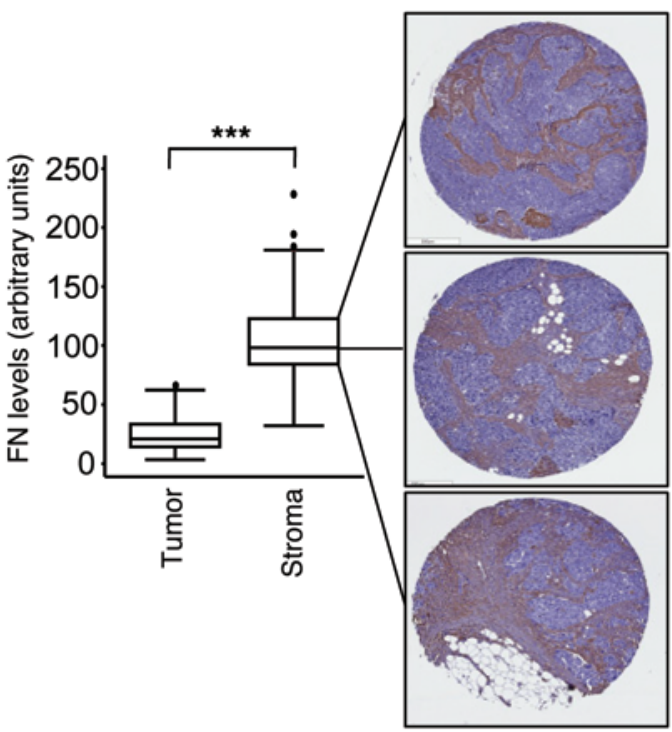

C
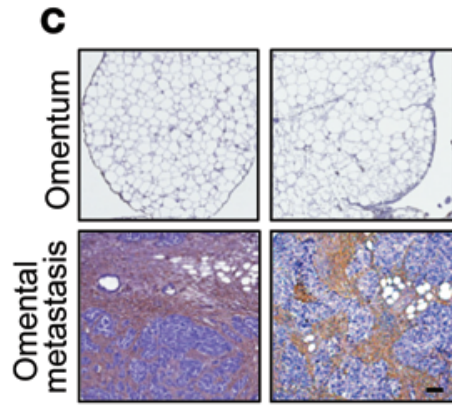

क्ञ̃. 100

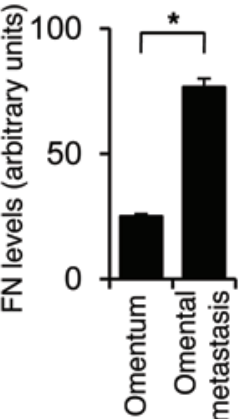

B

Omentum: Adhesion:

no tumor omental metastasis

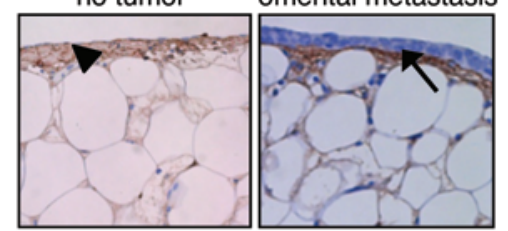

Adhesion/proliferation: omental metastasis

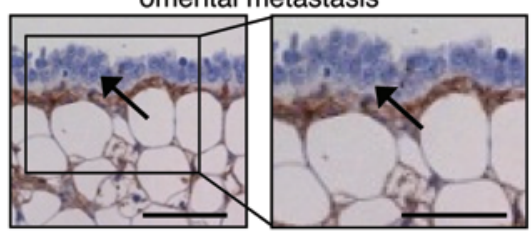

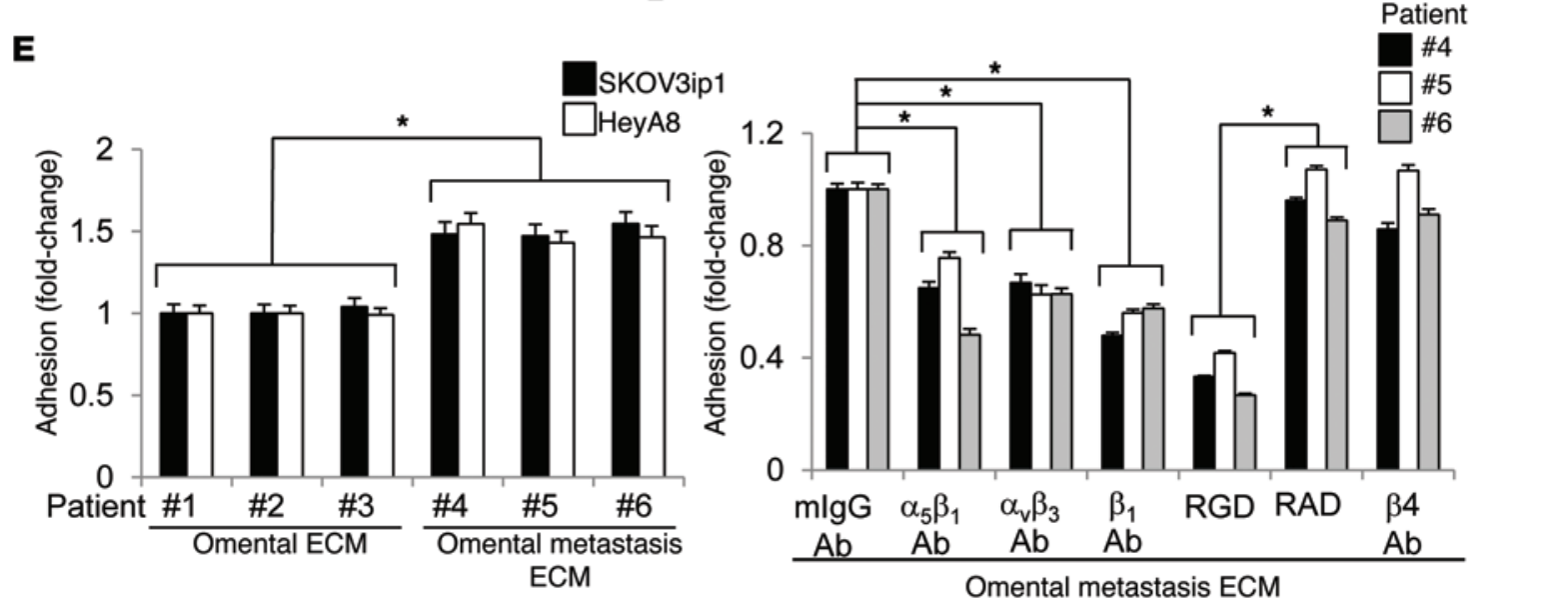

Figure 1. Fibronectin is overexpressed in the stroma of omental metastases. (A) Immunohistochemistry for fibronectin (FN) levels in the tumor and stromal compartments of omental metastases $(n=108)$ was analyzed in tumor sample cores using Aperio ImageScope and Spectrum software (see Supplemental Figure 9). Black dots, outliers; boxes, interquartile range (IQR); lines within boxes, median. ${ }^{* *} P<0.001$, Wilcoxon rank test (median \pm 1.5 IQR). 3 different tumor tissue cores from separate patients are shown. (B) Immunohistochemistry for fibronectin in tissue from a patient coincidentally detected with early, microscopic OvCa metastasis to the omentum (stage IIIA; representative sections of affected areas are shown). Arrowhead, mesothelial cells; arrows, OvCa cells. (C) Immunohistochemistry for fibronectin expression in omental tissues $(n=11)$ sampled from patients treated for benign disease and omental metastases $(n=43)$ removed from patients with serous papillary OvCa (mean \pm SEM). ${ }^{*} P<0.05$. (D) Immunoblot analysis of ECM extracted from omental tissues $(n=3)$ sampled from patients treated for benign disease and omental metastases removed from patients with serous papillary OvCa. rh-FN, recombinant human fibronectin. (E) Left: Adhesion (30 minutes) of OvCa lines SKOV3ip1 and HeyA8 to the isolated ECM in D. Right: Pretreatment of SKOV3ip1 cells for 30 minutes with integrin function blocking antibodies or peptides, followed by the adhesion assay to isolated ECM (mean \pm SEM; $n=5 ; 3$ independent experiments). mIgG, murine IgG control. ${ }^{*} P<0.05$, Student's $t$ test. Scale bars: $100 \mu$ m. 
A

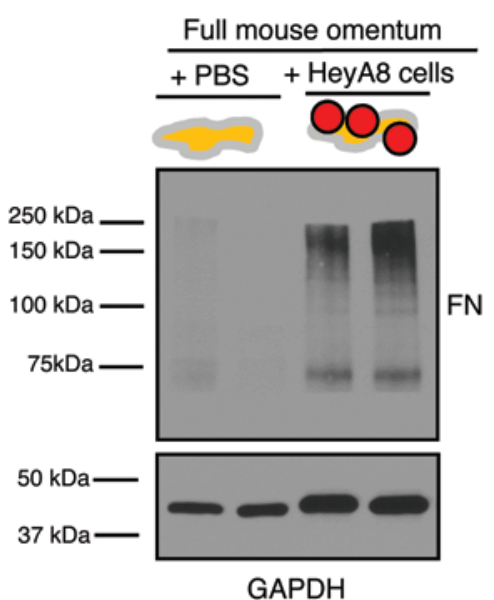

GAPDH
B

Cell lysates

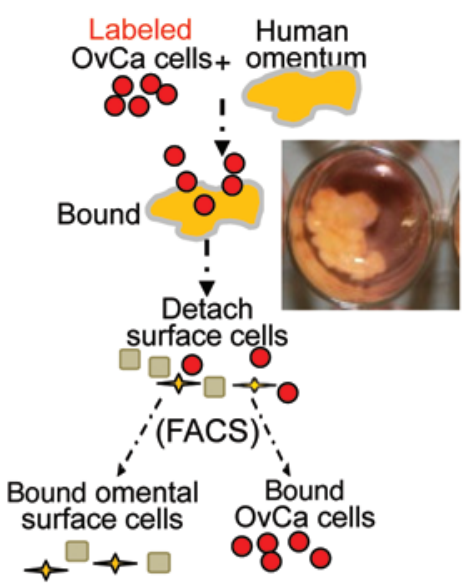

$250 \mathrm{kDa}$

$150 \mathrm{kDa}$

$100 \mathrm{kDa}$

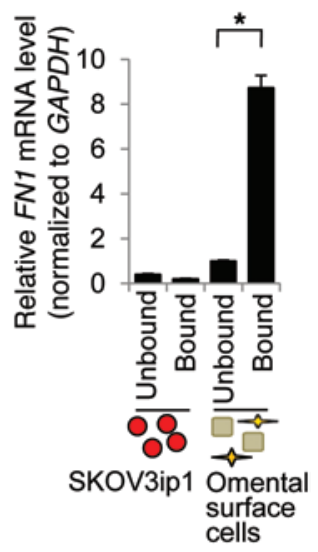

C

Fibronectin
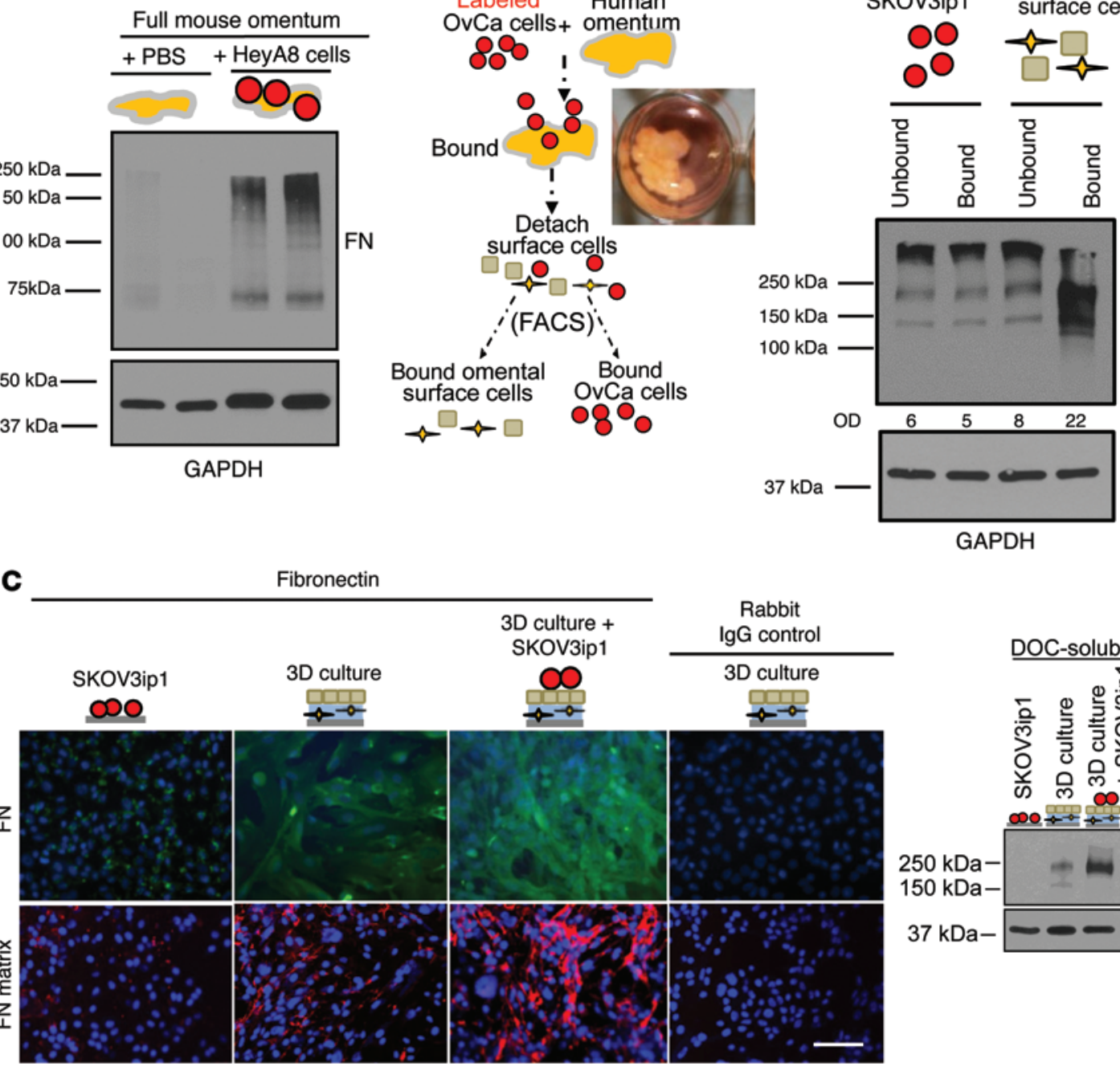
cells

D

Fibronectin matrix assembly

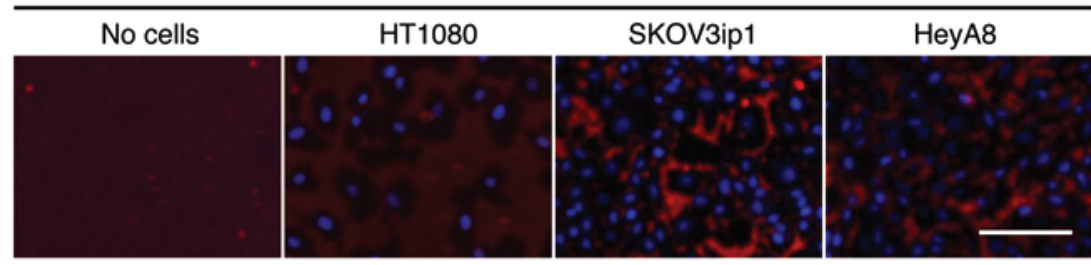

Rabbit

IgG control
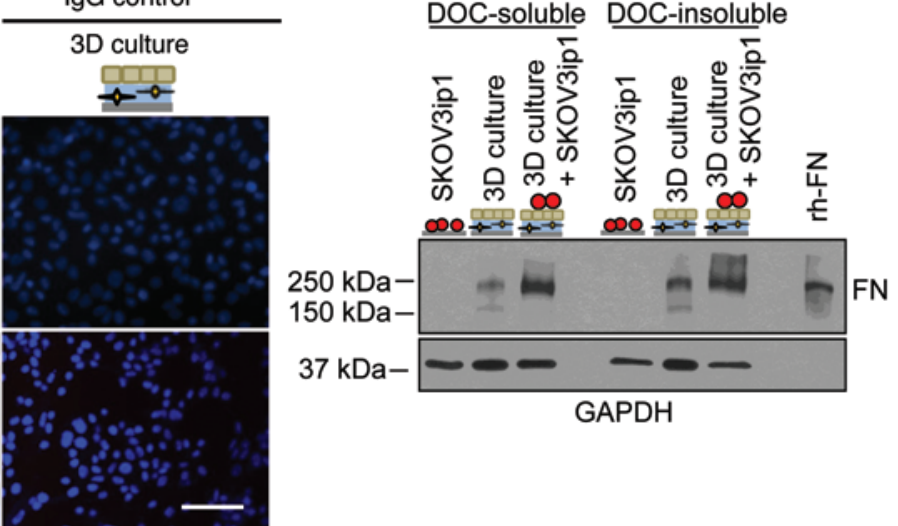

Figure 2. OvCa cells induce fibronectin matrix assembly in stromal cells. (A) Fibronectin protein expression in full mouse omentum after in vivo i.p. injection of HeyA8 OvCa cells for 48 hours. Immunoblot analysis was performed using a fibronectin-specific antibody. (B) Left: Ex vivo full human omentum adhesion, invasion, and proliferation assays with fluorescently labeled OvCa cells. Middle: Immunoblot analysis of fibronectin protein in the surface cells of the human omentum cultured alone (unbound) or with fluorescently labeled SKOV3ip1 cells (bound; after FACS). OD of fibronectin is shown normalized to GAPDH signal. Right: qRT-PCR analysis of FN1 mRNA expression. qRT-PCR was performed on mRNA using fibronectin specific probes (mean \pm SEM; $n=5$; 3 independent experiments). ${ }^{*} P<0.05$, Student's $t$ test. (C) Fibronectin protein production and matrix secretion were analyzed in the $3 D$ omental culture cocultured with SKOV3ip1 cells. Left: IF was performed using a fibronectin-specific antibody, and fibronectin production (green; IF with Triton-X 100) and fibronectin matrix secretion (red; IF without Triton-X 100) were analyzed. Right: Immunoblot analysis of fibronectin matrix production using DOCsoluble and -insoluble fractions of fibronectin (indicating a dense matrix) in SKOV3ip1 cells, 3D culture, or coculture. (D) Fibronectin matrix assembly by OvCa cells cultured on fibronectin. Fibronectin was seeded on a glass-bottomed plate, and SKOV3ip1, HeyA8, or HT1080 cells were added for 24 hours. IF for fibronectin (red) was performed; the formation of a dense fibronectin matrix was indicated by presence of fibrils. Scale bars: $50 \mu \mathrm{m}$. 

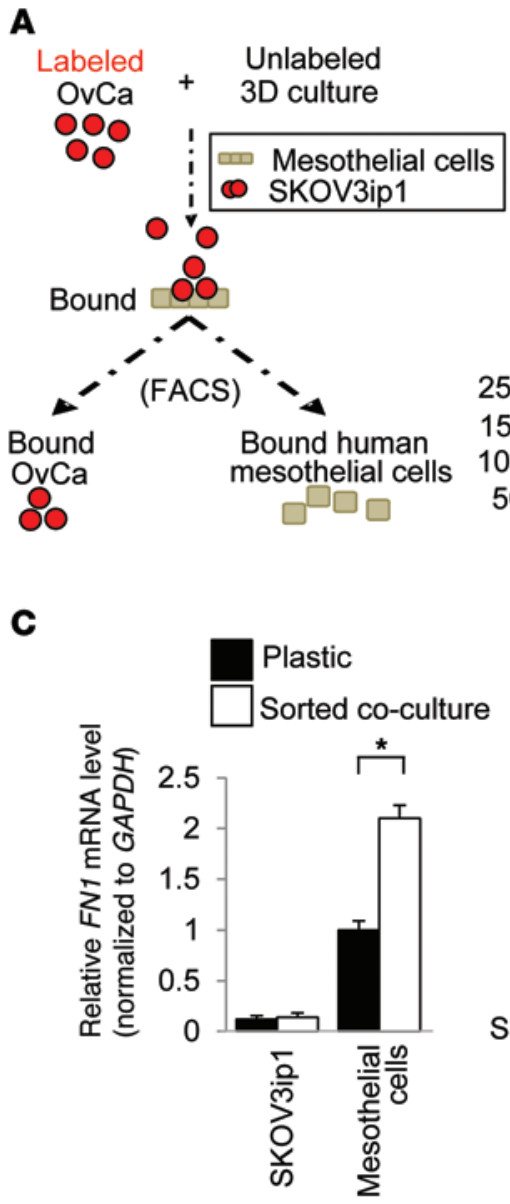
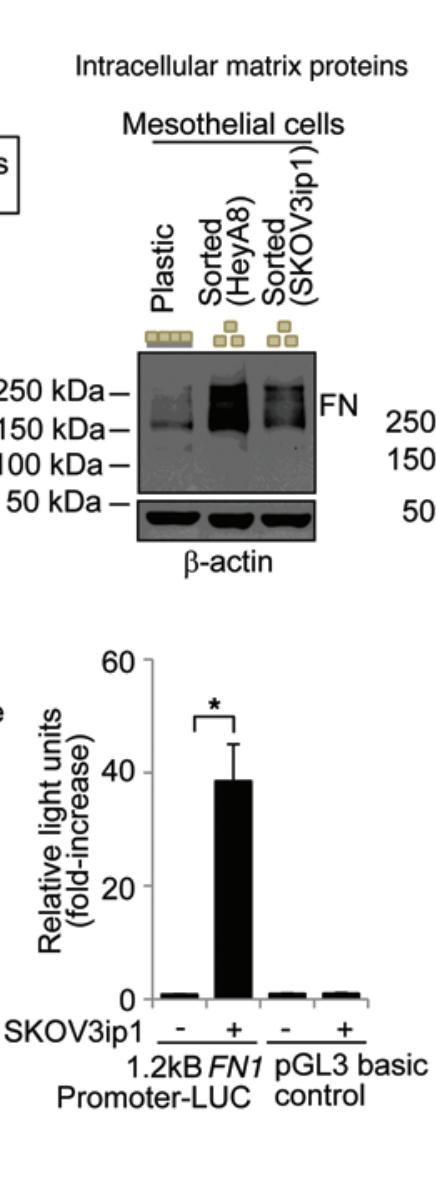

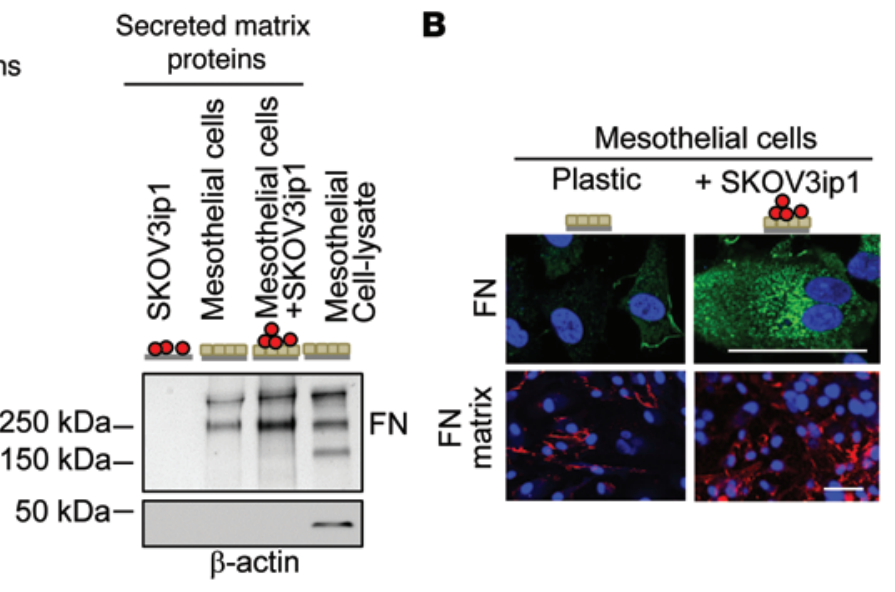

D

Cell lysates

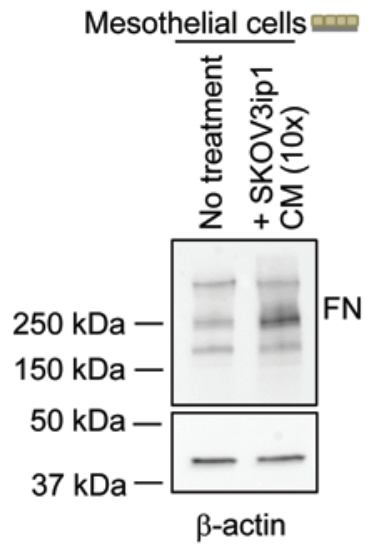

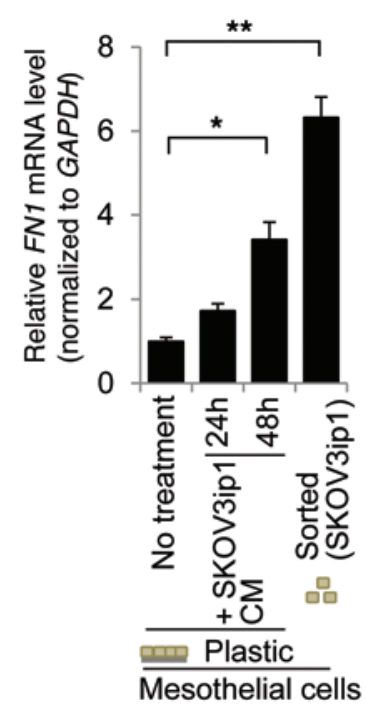

Figure 3. OvCa cells stimulate fibronectin expression in mesothelial cells. (A) Left: Coculture of fluorescently labeled OvCa cells and primary human mesothelial cells, followed by FACS. Middle: Immunoblot for fibronectin protein expression in cell lysates of primary human mesothelial cells grown on plastic or cocultured (48 hours) with the indicated OvCa cells, followed by separation using FACS. Right: Immunoblot for secreted levels of fibronectin (48 hours) in culture of SKOV3ip1 or primary human mesothelial cells on plastic, and after coculture. (B) IF analysis of fibronectin production (green) and fibronectin matrix secretion (red) in mesothelial cells cultured on plastic or cocultured with OvCa cells. (C) Left: FN1 mRNA expression. SKOV3ip1 or primary human mesothelial cells were cultured either on plastic or cocultured together (24 hours), followed by separation using FACS. mRNA was isolated, and qRT-PCR was performed using FN1-specific probes. Right: Primary human mesothelial cells were transfected with a luciferase reporter construct containing the full-length (1.2 kb) FN1 promoter (48 hours) and cultured on plastic or with SKOV3ip1 cells (24 hours). (D) Left: Immunoblot for fibronectin expression in primary human mesothelial cells after treatment with CM from SKOV3ip1 cells. Right: qRT-PCR analysis of FN1 mRNA expression in primary human mesothelial cells grown on plastic stimulated with or without SKOV3ip1 CM for 24 and 48 hours, or cocultured with SKOV3ip1 cells for 24 hours followed by separation of cells using FACS (mean \pm SEM; $n=5 ; 3$ independent experiments). ${ }^{*} P<0.05,{ }^{* *} P<0.01$, Student's $t$ test. Scale bars: $50 \mu$ m.

fibronectin production. Fluorescently labeled OvCa cells were cocultured with primary human mesothelial cells for 48 hours, then separated by FACS. The cocultured OvCa cell lines strongly induced both intracellular and secreted fibronectin in the mesothelial cells, which laid down a dense fibrillar fibronectin matrix, whereas the cell lines cultured alone secreted a negligible amount of fibronectin (Figure 3, A and B). The OvCa cell-mediated fibronectin induction was paralleled by FN1 mRNA induction in the mesothelial cells (Figure 3C), suggestive of transcriptional regulation of fibronectin upon interaction with the OvCa cells. Indeed, transfection of primary human mesothelial cells with the full-length FN1 promoter (28), followed by cocultivation with SKOV3ip1 cells, increased FN1 promoter activity almost 40-fold
(Figure 3C). Fibronectin induction in mesothelial cells was not limited to direct OvCa cell/mesothelial cell interaction, since CM from the OvCa cells was able to induce both protein and mRNA expression of fibronectin (Figure 3D). CM from the high-grade serous OvCa cell lines Kuramochi and Tyk-nu, as well as from RKO colon cancer cells, induced fibronectin in mesothelial cells (Supplemental Figure 3, A and B). The mesothelial cell response to cancer cells was not limited to omental tissue-derived mesothelial cells. Both peritoneal wall- and fallopian serosa-derived mesothelial cells increased fibronectin production when treated with OvCa cell CM (Supplemental Figure 3, C and D).

Next, we inhibited fibronectin production (Figure 4A) and secretion (Supplemental Figure 4 and Supplemental Methods) 
A

Human mesothelial cells
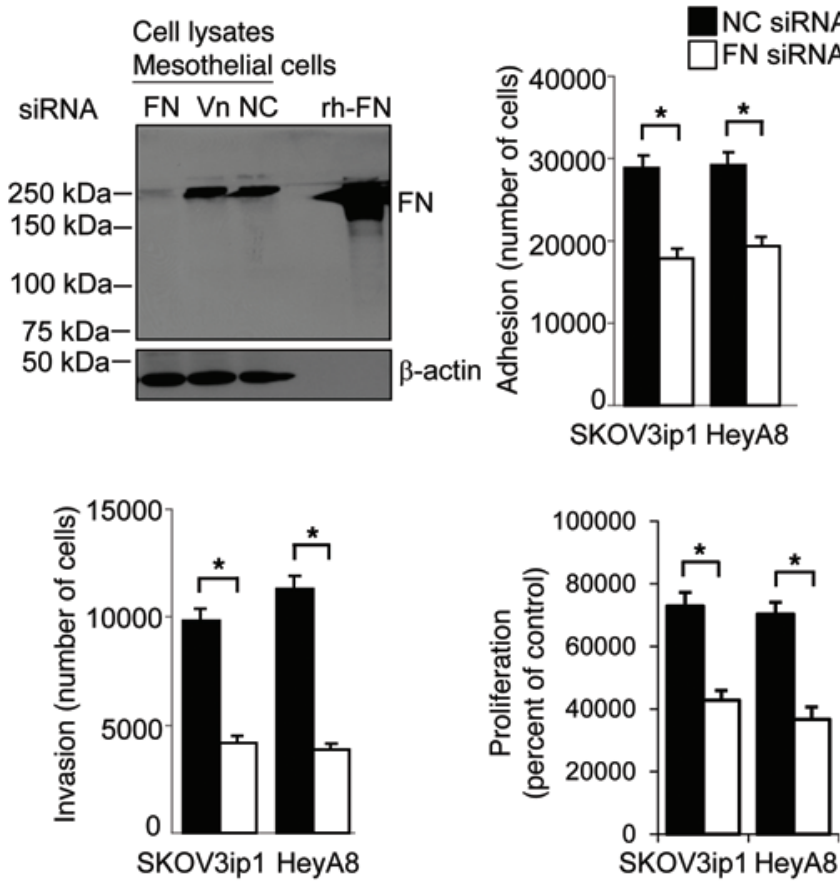

B

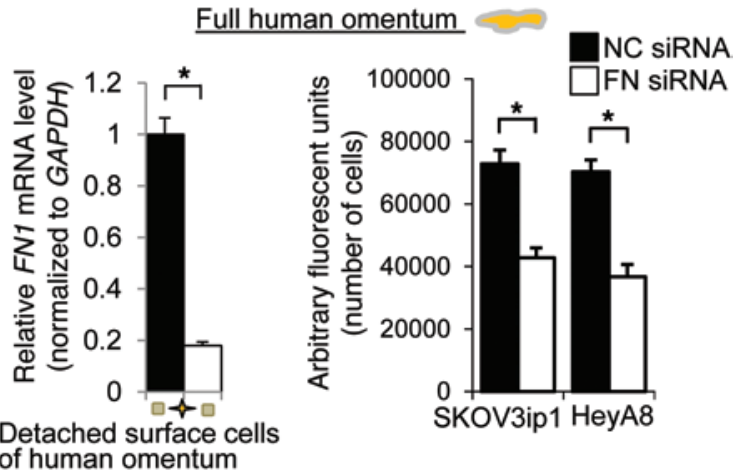

Figure 4. Inhibition of fibronectin in mesothelial cells or omentum impairs OvCa cell adhesion, invasion, and proliferation. (A) Fibronectin knockdown in primary human mesothelial cells. Immunoblot analysis of fibronectin in primary human mesothelial cells transfected with fibronectin-specific (FN), vitronectin-specific $(V n)$, or control (NC) siRNA. Functional assays investigating the effect of fibronectin knockdown in mesothelial cells on OvCa cell adhesion (30 minutes), invasion

(24 hours), and proliferation (96 hours). Primary human mesothelial cells were transfected with fibronectin-targeted or control siRNA, followed by addition of fluorescently labeled SKOV3ip1 and HeyA8 OvCa cells, which were detected using a fluorescence reader (mean $\pm \mathrm{SEM} ; n=5$ [adhesion and proliferation], 3 [invasion]; 3 independent experiments).

${ }^{*} P<0.05$, Student's $t$ test. (B) Fibronectin knockdown in pieces of full human omentum. Left: Transfection of fibronectin siRNA (specifically designed for in vivo use) in pieces of full human omentum (72 hours); FN1 mRNA downregulation was confirmed in detached surface cells (after scraping off surface cells of the omentum) using qRT-PCR. Right and bottom: Adhesion, invasion, and proliferation of OvCa cells was inhibited on full human omentum when fibronectin expression in omental surface cells decreased (mean $\pm \mathrm{SEM} ; n=5$; 3 independent experiments). ${ }^{*} P<0.05$, Student's $t$ test. in primary human mesothelial cells using siRNA and evaluated adhesion, invasion, and proliferation of 2 OvCa cell lines. Without the fibronectin support from the mesothelial cells, neither of the OvCa cell lines were able to adhere (59\% of control), invade $(36 \%)$, or proliferate (54\%) as efficiently (Figure $4 \mathrm{~A})$. Furthermore, when fibronectin was knocked down in the mesothelial cells that covered a full piece of human omentum, the OvCa cells were again not able to adhere, invade, or proliferate as efficiently as control transfected cells (Figure 4B).

These experiments were corroborated in vivo using a transgenic mouse model of fibronectin deficiency. Homozygous floxed fibronectin 1 (Fn1) mice ( $F n 1^{f / f l}$ mice) do not have an obviously abnormal phenotype, and have normal fertility and life spans. However, upon induction of ischemic brain injury, the deletion of Fn1 leads to large brain infarcts, which are, at least in part, due to the absence of the antiapoptotic function of fibronectin (29). Using the $F n 1^{f / f l}$ mice, we endeavored to determine the role of fibronectin during early metastasis in vitro and in vivo. Primary resting omental mouse mesothelial cells established from $F n 1^{f / f l}$ mice were infected with Cre recombinase-containing adenovirus (referred to herein as Ad-Cre) to delete Fn1. Because resting mouse mesothelial cells (Figure 2A), like their human counterparts (Figure 3A), did not constitutively express fibronectin, they were stimulated with TGF- $\beta$, a known inducer of fibronectin expression (30). Deletion of fibronectin abrogated TGF- $\beta$-mediated Fn1 mRNA induction (Figure 5A and Supplemental Figure 5), confirming successful $F n 1$ deletion. When the primary $F n 1^{f / A}$ mesothelial cells were cocultured with ID8 mouse OvCa cells stably expressing GFP, adhesion, invasion, and proliferation were significantly impaired, at levels $60 \%, 60 \%$, and $74 \%$, respectively, of the empty adenovirus control (Ad-Cont) (Figure 5A). Next, we deleted fibronectin expression in vivo on the surface cells lining the abdominal cavity through i.p. injection of Ad-Cre into $F n 1^{f / f}$ mice. Since the abdominal cavity and organs are covered by mesothelial cells (2, $25,27)$, i.p. injection of Ad-Cre will delete fibronectin expression, primarily in the mesothelial cells. Quantitative real-time RT-PCR (qRT-PCR) analysis 2 days after Ad-Cre injection showed successful in vivo knockdown of fibronectin (Figure 5B). Compared with Ad-Cont-injected mice, Ad-Cre-pretreated $F n 1^{f / f l}$ mice injected i.p. with GFP-expressing ID8 mouse OvCa cells yielded fewer omental metastases (Figure 5, C and D). Furthermore, treatment of GFP-expressing ID8 cells with an $\alpha_{5}$ integrin-specific antibody inhibited OvCa metastasis by $52 \%$ in the $F n 1^{f / f l}$ mice, while knocking out fibronectin with Ad-Cre treatment in the $F n 1^{f / f}$ mice reduced OvCa metastasis by 76\%. Combination therapy with $\alpha_{5}$ integrin antibody and Ad-Cre treatment in the $F n 1^{f / f l}$ mice inhibited OvCa metastasis by $86 \%$. These data suggest that the prometastatic function of fibronectin involves a non-fibronectin-receptormediated factor. On the basis of the combined data in Figures 4 and 5 , we propose that mesothelial cell fibronectin expression promotes early OvCa metastasis.

Tumor cells induce fibronectin expression in mesothelial cells through a TGF- $R$ RI/RAC1/SMAD3-dependent signaling pathway. Analysis of gene expression data from the Australian Ovarian Cancer Study (AOCS) and from the Cancer Genome Atlas (TCGA) OvCa data showed that FN1 mRNA was most highly expressed in the $\mathrm{C} 1$ molecular subtype (high stromal response; Supplemental 
A Mesothelial cells from Fn1 ${ }^{n / H}$ mice

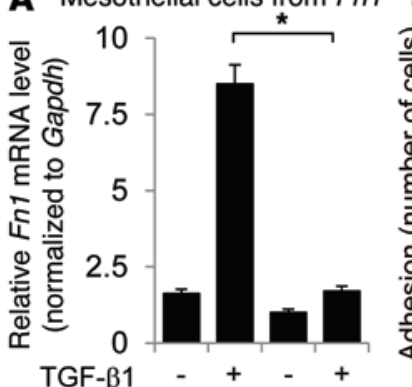

Treatment $\overline{\text { Ad-Cont }} \overline{\text { Ad-Cre }}$

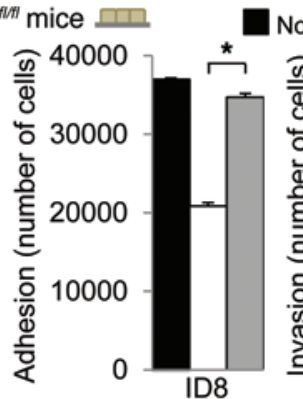

No treatment $\square$ Ad-Cre

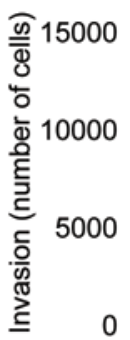

0

ID8

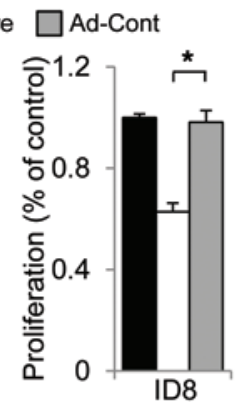

ID8

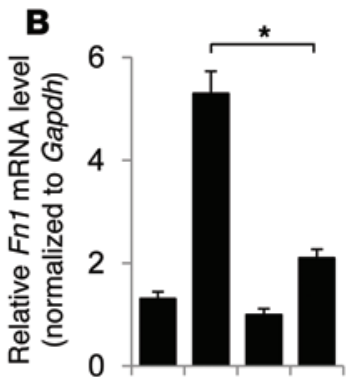

ID8 cells $-++\quad+$ treatment $\overline{\text { Ad-Cont }} \overline{\text { Ad-Cre }}$

Detached surface cells of mouse omentum
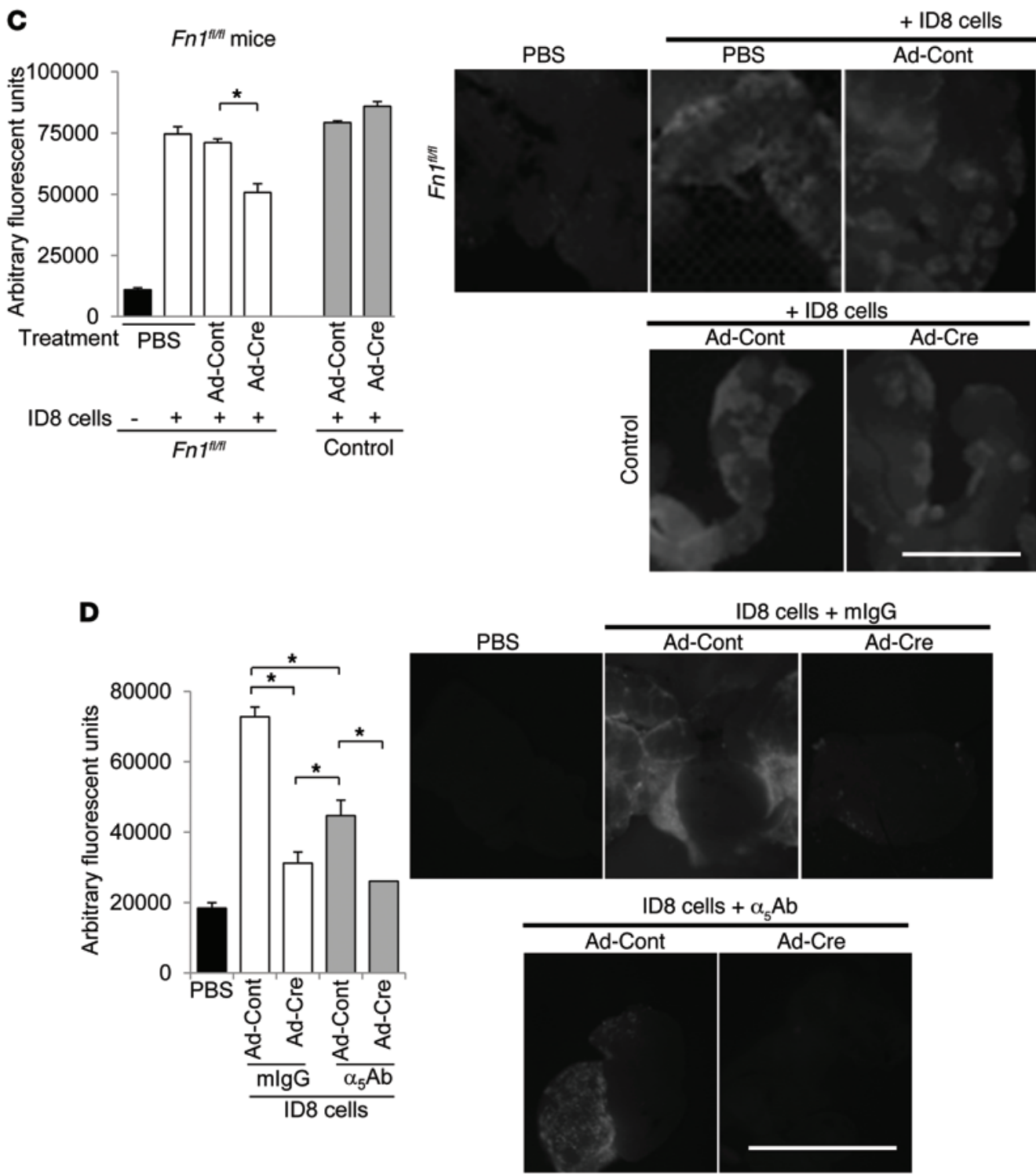

Figure 5. Genetic knockdown of $\boldsymbol{F n} 1$ reduces metastasis. (A) Left: qRT-PCR analysis for Fn1 on mRNA isolated from mouse mesothelial cells cultured from $F n 7^{f / f l}$ mice treated for 72 hours with Ad-Cont or Ad-Cre, followed by treatment with TCF- $\beta 1$ for 24 hours. Right: Functional assays investigating the effect of fibronectin knockdown in primary Fn $7^{\mathrm{fl} / \mathrm{f}}$ mesothelial cells on ID8 mouse OvCa cell adhesion, invasion, and proliferation. (B-D) In vivo knockdown of fibronectin in omental surface cells of $F n 7^{f / f l}$ mice. Ad-Cre or Ad-Cont was injected i.p. into Fn $7^{f / f}$ mice for 96 hours to downregulate fibronectin, followed by i.p. injection of $4 \times 10^{6} \mathrm{GFP}$-expressing ID8 OvCa cells. (B) 48 hours after injection, the mouse omentum was removed and digested, omental surface cells were collected by FACS, and qRT-PCR was performed for Fn1 on mRNA isolated from the cells. (C and D) Metastasis assay. (C) Fn7fl/fl or control C57BL/6 mice were injected with PBS, Ad-Cre, or Ad-Cont. (D) GFP-expressing ID8 cells were mixed with control lgG or $10 \mu \mathrm{g} / \mathrm{ml}$ murine $\alpha_{5}$ integrin antibody for 15 minutes prior to i.p. injection. 72 hours after ID8 cell injections, the mouse omentum was removed, imaged using fluorescent microscopy (right), and digested, and cancer cells were quantified using a fluorescence reader. Data are mean \pm SEM $\left(n=3\right.$ [invasion and qRT-PCR assays] or 5 [other assays]). ${ }^{*}<0.05$, Student's $t$ test. Scale bars: 2 mm. 
A

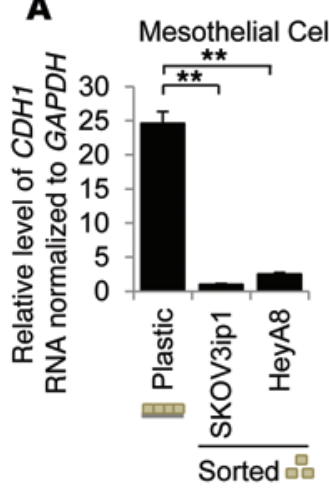

B

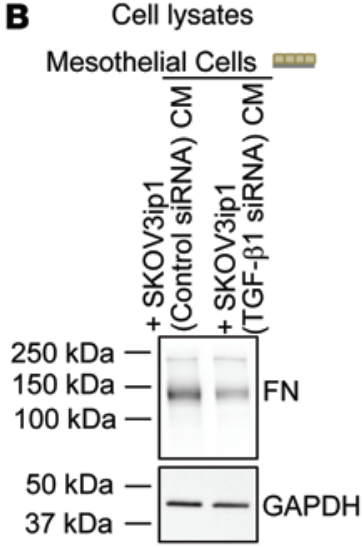

Cell lysates

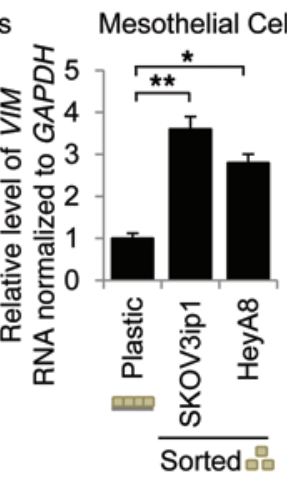

s
Mesothelial Cells

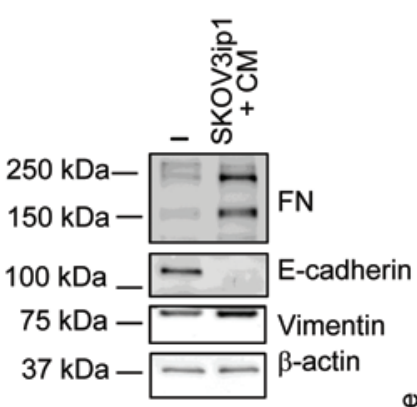

孪

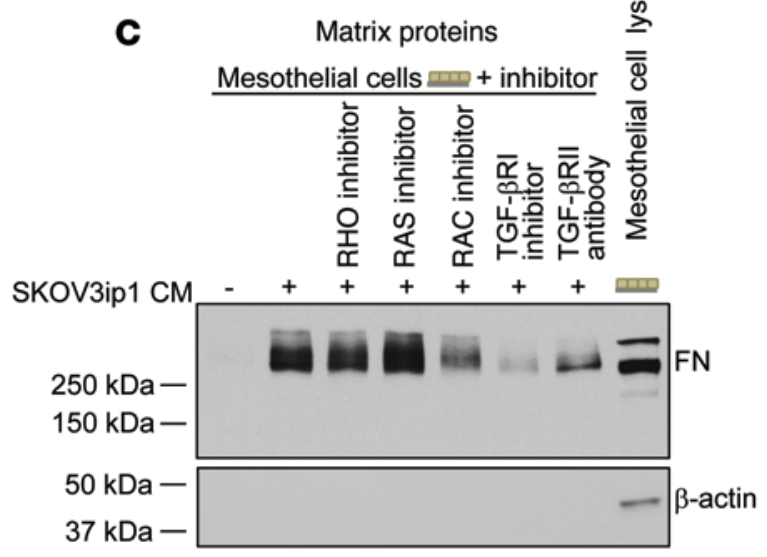

Figure 6. Tumor cells induce fibronectin expression in mesothelial cells through a TGF-ß1-dependent signaling pathway. (A) Left: qRT-PCR analysis for $C D H 1$ and VIM. Primary human mesothelial cells were cultured on plastic or cocultured with the indicated OvCa cells for 48 hours followed by cell separation using FACS and extraction of RNA (mean \pm SEM; $n=3 ; 3$ independent experiments). ${ }^{*} P<0.05$, ${ }^{* *} P<0.01$, Student's $t$ test. Right: Immunoblot analysis of fibronectin, E-cadherin, and vimentin in primary human mesothelial cells treated with or without SKOV3ip1 CM. (B and C) Immunoblots for fibronectin. (B) Primary human mesothelial cells were treated with SKOV3ip1 CM for 72 hours, and cell lysates were extracted. (C) Detection of fibronectin secreted by primary human mesothelial cells treated with or without SKOV3ip1 CM and the RHO kinase inhibitor, the RAS signaling activity inhibitor, the RAC-CEF interaction inhibitor, the TCF- $\beta$ RI kinase inhibitor I, or the TGF- $\beta$ RII blocking antibody.

was abrogated with RAC1 inhibitor treatment in SKOV3ip1 cocultured mesothelial cells (Supplemental Figure 7A). Consistent with a prominent role for TGF- $\beta 1$ signaling in mesothelial cells, coculture of the 2 cell types showed strong induction of

Figure 6A), which is associated with serous high-grade cancer and the poorest survival (31). Subsequent pathway analysis indicated that the epithelial to mesenchymal transition (EMT) was the second most significant biological pathway that correlated with elevated fibronectin expression in the AOCS and TCGA cohorts (Supplemental Tables 1 and 2 and ref. 31). This bioinformatic analysis was then tested experimentally. Addition of SKOV3ip1 CM, or coculture of the cancer cells with primary mesothelial cells, led to decreased E-cadherin and increased vimentin protein and mRNA expression in the mesothelial cells (Figure 6A and Supplemental Figure 6B). Additionally, OvCa CM induces EMT morphology in the mesothelial cells (Supplemental Figure 6, C and $\mathrm{D}$, and ref. 32 ). We conjectured that cancer cell-derived TGF- $\beta 1$ mediates fibronectin production and/or secretion in mesothelial cells, because (a) TGF- $\beta 1$-dependent EMT pathways correlated with fibronectin expression (Supplemental Table 2), (b) TGF- $\beta 1$ induced EMT in mesothelial cells (Supplemental Figure 6, C and D, and ref. 33), (c) SKOV3ip1 cells secreted TGF- $\beta 1$ (Supplemental Figure 6E), and (d) TGF- $\beta 1$ is highly expressed in omental metastasis (34). To test this hypothesis, TGF- $\beta 1$ expression was inhibited in SKOV3ip1 cells using siRNA. CM from these cells induced less fibronectin production in mesothelial cells than did CM from control siRNA-stimulated cells (Figure 6B and Supplemental Figure 6F). SKOV3ip1 CM-stimulated mesothelial cells were then treated with an inhibitor of TGF- $\beta$ RI, an antibody against TGF- $\beta$ RII, and inhibitors of small $\mathrm{G}$ proteins (the downstream TGF- $\beta 1$ effectors RAC, RAS, and RHO; ref. 35). The TGF- $\beta$ RI inhibitor, the TGF$\beta$ RII antibody, and the RAC inhibitor blocked fibronectin secretion by mesothelial cells; however, the RAS and RHO inhibitors had no such effect (Figure 6C). Comparably, FN1 mRNA expression phosphorylated SMAD2/3 (36) in mesothelial cells, as exhibited by immunoblot after FACS and immunofluorescence (IF) (Figure 7A). When we inhibited SMAD3 using siRNA in mesothelial cells or added a TGF- $\beta$ RI inhibitor or TGF- $\beta$ RII antibody to mesothelial cells followed by the addition of SKOV3ip1 CM, fibronectin secretion was impaired (Figure 7B), which was paralleled by reduced FN1 mRNA in the mesothelial cells (Supplemental Figure 7A).

Coculture of OvCa cells with mesothelial cells strongly induced RAC1 activity, while total RAC1 expression was unchanged (Figure 7C and Supplemental Figure 7B). The increase in RAC1-GTP was specifically observed (by IF) in mesothelial cells after coculture with SKOV3ip1 cells (Figure 7C). Inhibition of RAC1 expression in mesothelial cells with either a RAC1 siRNA or the RAC1 inhibitor abrogated FN1 mRNA in cocultured mesothelial cells and fibronectin secretion in OvCa cell CM-stimulated mesothelial cells (Figure 7D and Supplemental Figure 7A). Because our findings suggested that both RAC1 and TGF- $\beta 1$ signaling regulate fibronectin expression, we asked whether TGF- $\beta 1$ regulates fibronectin through RAC1 signaling. Indeed, the TGF- $\beta 1-$ mediated induction of fibronectin expression in mesothelial cells could be abrogated by blocking RAC1 signaling (Figure 7E). Taken together, these data suggest that tumor cells induce EMT in mesothelial cells through TGF- $\beta 1$ signaling.

Blocking $\alpha_{5}$ integrin or $\beta_{1}$ integrin prevents OvCa metastasis. In view of the critical role of fibronectin during early OvCa metastasis, 2 potential clinically applicable fibronectin receptor function blocking antibodies were evaluated in vitro and in vivo. The established OvCa cell lines HeyA8 and SKOV3ip1 were fluorescently labeled and then cultured on the 3D culture with antibodies. Both $\alpha_{5}$ integrin (37) and $\beta_{1}$ integrin (38) blocking antibodies prevented 
A
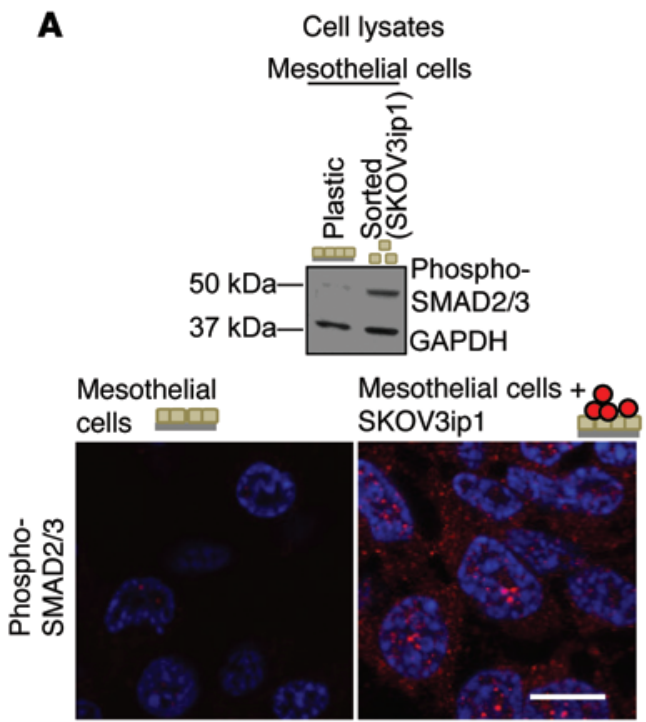

C

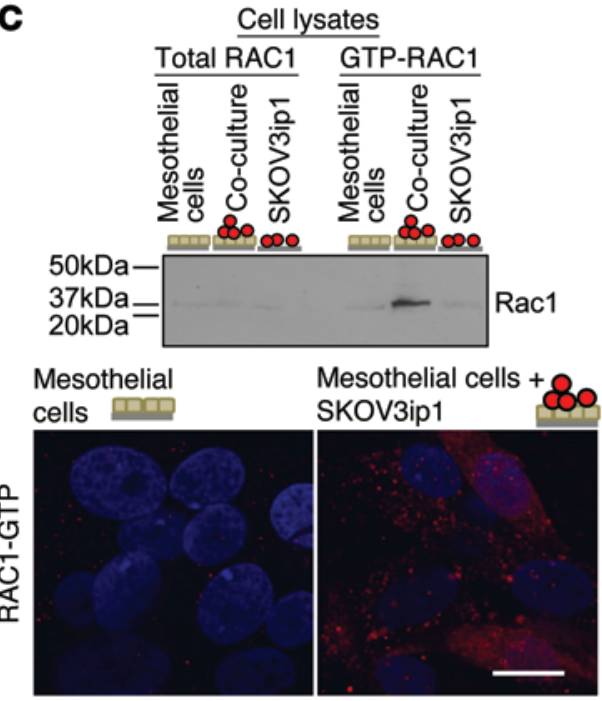

B
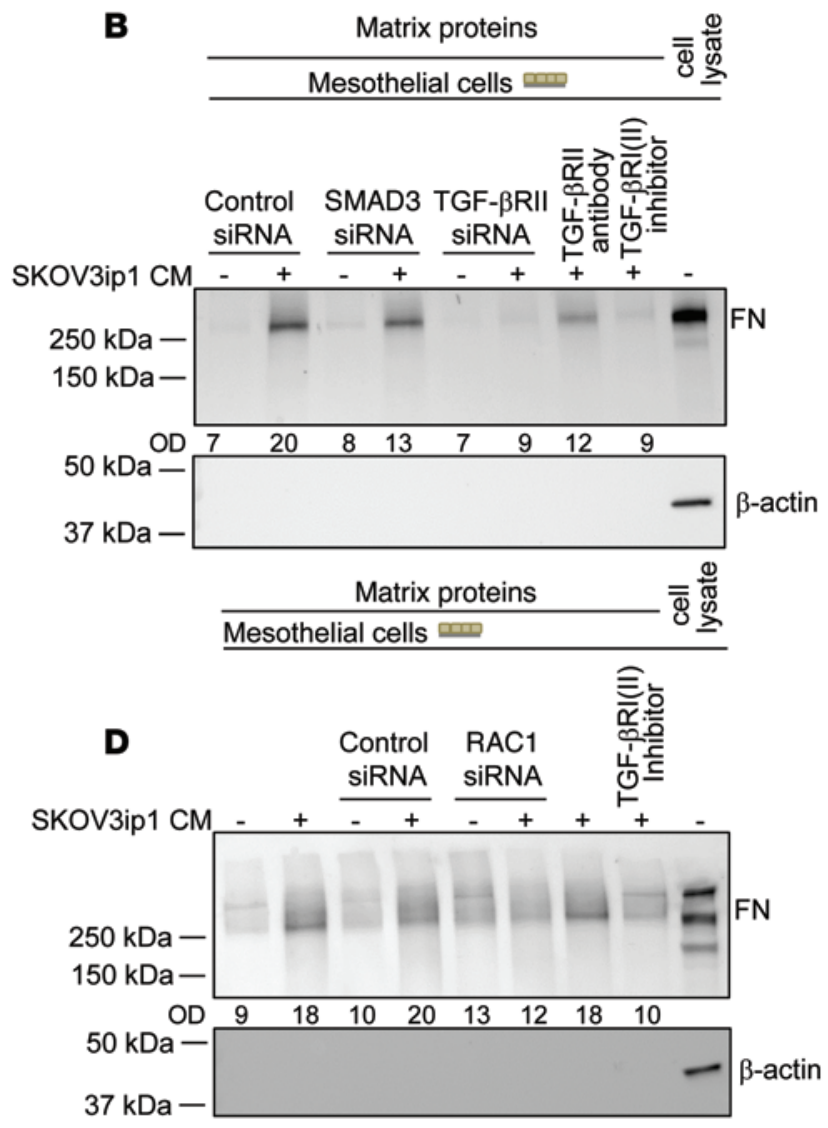

E

Cell lysates

Mesothelial cells

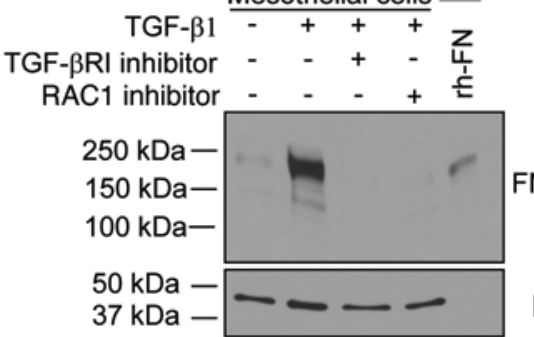

FN

$\beta$-actin

Figure 7. OvCa cells regulate fibronectin expression in mesothelial cells by a RAC1/SMAD3-dependent signaling pathway. (A) Immunoblot and IF analysis using a phospho-SMAD2/3 antibody in primary human mesothelial cells cultured on plastic or cocultured with SKOV3ip1 cells. The primary human mesothelial cells were separated from SKOV3ip1 cells using FACS (top). (B) Immunoblot analysis for secreted fibronectin. Primary human mesothelial cells were transfected with control, SMAD3, or TCF- $\beta$ RII siRNA, followed by treatment with or without SKOV3ip1 CM and the TCF- $\beta$ RII antibody or the TCF- $\beta$ RI kinase inhibitor II for 72 hours. OD of fibronectin is shown normalized to total protein signal. (C) Top: RAC1 activity assay. Immunoblot analysis for total and active RAC1 (pulldown with a GTP-specific antibody) in cell lysates of primary human mesothelial cells, SKOV3ip1 cells, or coculture. Bottom: IF of RAC1-GTP in mesothelial cells cultured on plastic or cocultured with SKOV3ip1 cells for 24 hours using antibody specific for RAC1-GTP. (D) Immunoblot analysis for fibronectin secreted by primary human mesothelial cells transfected with RAC1 siRNA, followed by treatment with SKOV3ip1 CM and TCF- $\beta$ RI kinase inhibitor II. OD of fibronectin is shown normalized to total protein signal. (E) Immunoblot analysis for fibronectin in cell lysates of primary human mesothelial cells treated in combination with TCF- $\beta 1$, TCF- $\beta$ RI kinase inhibitor I, and/or a RAC1 inhibitor for 48 hours. Scale bars: $10 \mu \mathrm{m}$.

in vitro adhesion by an average of $60 \%$ and invasion and proliferation by an average of $40 \%$ (Figure $8 \mathrm{~A}$ ). To study the in vivo efficacy of the 2 antibodies, we tested them in a prevention study (treatment the day of and 2 and 4 days after OvCa cell intraovarian injection) that mimicked treatment during initiation of metastasis. Pretreatment of mice with $\alpha_{5}$ integrin or $\beta_{1}$ integrin blocking antibodies reduced metastasis number and tumor weight by more than $90 \%$ compared with IgG control-treated mice (Figure 8B). Finally, the therapeutic effect of a compound now in clinical testing, ATN-161 (Ac-PHSCN-NH2), a 5-mer capped peptide derived from the fibronectin synergy region, was evaluated in vitro and in vivo. The synergy region of fibronectin is a ligand for $\alpha_{5} \beta_{1}$ integrin, which increases the avidity of $\alpha_{5} \beta_{1}$ integrin for the fibronectin RGD sequence (19). The peptide blocked both proliferation and invasion of the established OvCa cell lines HeyA8 and SKOV3ip1 by an average of $29 \%$ and $52 \%$, respectively (Supplemental Figure 8A). To study the in vivo efficacy of ATN-161, we took 2 approaches (37): (a) testing the peptide in a prevention study 
A

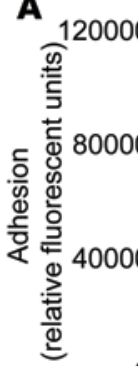

0 SKOV3ip1 HeyA8

B
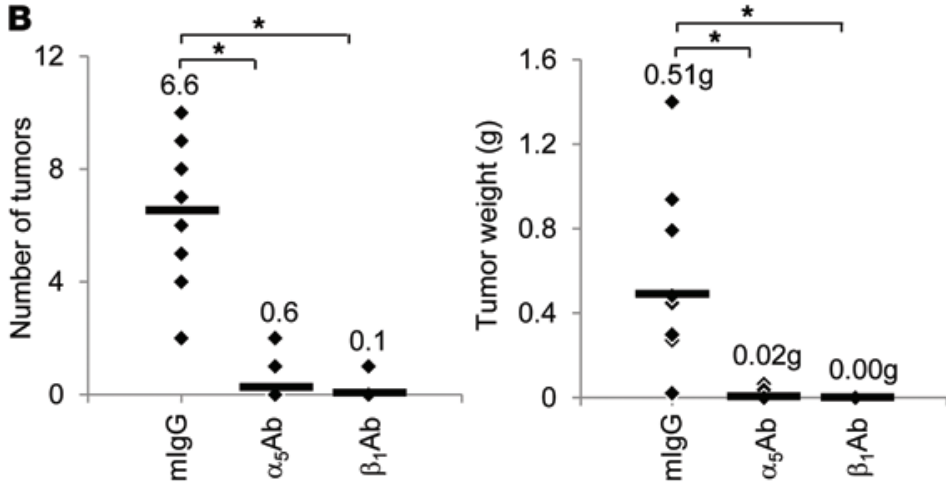

C

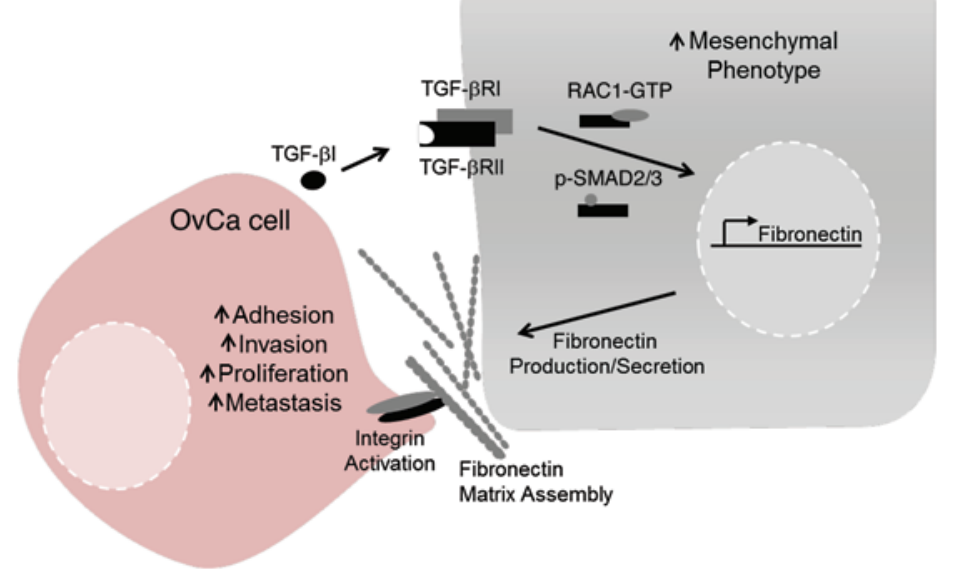

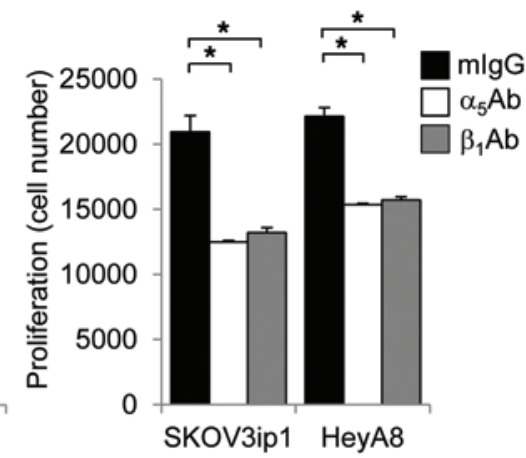

Figure 8. Blocking $\alpha_{5}$ or $\beta_{1}$ integrin prevents OvCa metastasis. (A) In vitro testing of $\alpha_{5}$ and $\beta_{1}$ integrin blocking antibodies. OvCa cell lines HeyA8 and SKOV3ip1 were mixed with antibodies $(10 \mu \mathrm{g} / \mathrm{ml})$ for 15 minutes, and adhesion ( 2 hours), invasion (24 hours), and proliferation (96 hours) to the $3 \mathrm{D}$ omental culture were measured (mean \pm SEM; $n=3$ [invasion] or 5 [adhesion and proliferation]; 3 independent experiments). ${ }^{*} P<0.01$. (B) In vivo testing of $\alpha_{5}$ and $\beta_{1}$ integrin blocking antibodies using the HeyA8 xenograft model of OvCa metastasis. HeyA8 cells were mixed with antibodies $(10 \mu \mathrm{g} / \mathrm{ml})$ and injected into the ovary $\left(2.5 \times 10^{4}\right)$. Mice were treated 2 and 4 days after inoculation with i.p. antibody injection $(10 \mathrm{mg} / \mathrm{kg})$, and the number of metastatic tumors (left) and tumor weight (right) were determined 28 days after injection. Shown are individual data points and means of treatment groups (horizontal lines and numeric values). ${ }^{*} P<0.01$, Student's $t$ test. (C) OvCa and mesothelial cell communication during the initial steps of OvCa metastasis (see text for details). (treatment the day of and 3 and 5 days after OvCa cell i.p. injection) that mimicked treatment of microscopic disease, and (b) treating advanced tumors in an intervention study (treatment 3 times per week starting on day 7 after OvCa cell i.p. injection), a design that mimicked treatment of advanced disease. Pretreatment of mice with ATN-161 in the prevention study reduced metastasis number by $52 \%$ and tumor weight by $70 \%$ compared with control-treated mice (Supplemental Figure 8B). Surprisingly, the peptide also reduced metastatic spread by $66 \%$ and tumor weight by $65 \%$ in the intervention study, when the drug was given after the establishment of tumors (Supplemental Figure 8C). Taken together, these data suggest that inhibiting the interaction of integrins with fibronectin blocks both early and late steps of OvCa metastasis.

\section{Discussion}

In recent years, we have come to understand that stromal cells in the tumor microenvironment and the ECM are important com- ponents of the metastatic process (10). However, the role of these cells in the molecular events underlying the early dissemination of abdominally metastasizing tumors remains poorly defined. The peritoneal cavity and the omentum are lined by the mesothelium, a monolayer of mesothelial cells resting on a basement membrane. This tissue functions to maintain serosal integrity and provide a nonadhesive surface that allows smooth bowel movements. It also acts as a permeability barrier, secreting various substances involved in the regulation of peritoneal permeability and local host defense (2). By testing several stromal cell components using an organotypic 3D model of the human omentum/peritoneum that we developed previously $(25,39)$, we found here that OvCa cells specifically induced protein expression, mRNA production, and promoter activity of fibronectin in mesothelial cells (Figures 2 and 3). Conversely, by inhibiting fibronectin expression in the mesothelial cells using siRNA, we were able to reduce adhesion, migration, and invasion in vitro (Figure 4), and by using an 
inducible floxed fibronectin mouse model, we reduced metastasis in vivo (Figure 5). These results suggest that OvCa cells recruit mesothelial cells, inducing them to secrete fibronectin to help the cancer cells establish the initial metastatic colony (Figure $8 \mathrm{C}$ ). Indeed, the behavior of the primary mesothelial cells we studied suggested that they become associated with cancer cells (i.e., cancer-associated mesothelial cells [CAMs]) in a manner analogous to CAFs (40) and cancer-associated adipocytes $(15,41)$. This supports a broader concept that cancer cells are able to recruit a variety of cell types in the microenvironment to create an activated tumor stroma that supports growth and metastasis. More than $20 \%$ of OvCas present as stage IV with pleural effusion, but without radiologic evidence of intrapulmonary nodules. Although we did not study pleural metastasis, it is interesting to note that the pleural cavity is also lined by a single layer of mesothelial cells. This raises the possibility that a similar mechanism contributes to colonization of the pleura in OvCa.

In the presence of OvCa cells secreting TGF- $\beta 1$, mesothelial cells underwent EMT, upregulating vimentin and downregulating E-cadherin. Their phenotype then changed from a cobblestone pattern to one of spindle-like cells resembling fibroblasts (Figures 6 and 7). Secretion of fibronectin is a well-known mesenchymal marker that was previously used to confirm EMT in mesothelial cells $(2,42)$. In mesothelial cells cocultured with OvCa cells, TGF- $\beta 1$ bound to the TGF- $\beta$ RI and activated a RAC1/ SMAD-dependent signaling pathway that strongly induced the transcriptional upregulation of fibronectin (Figures 6 and 7), thus promoting the metastatic cascade. A similar mechanism has also been described for keratinocytes, in which TGF- $\beta 1$ downregulates E-cadherin and upregulates a RAC-dependent signaling pathway $(43,44)$. This is consistent with our present results that RAC1 activity was upregulated when mesothelial cells and OvCa cells were cocultured.

Activation of an EMT program, a general physiological response of the mesothelium to stress, is a phenomenon reported during peritoneal dialysis and peritoneal inflammation. Peritoneal irritation caused by peritoneal dialysis induces mesothelial cells to transition from an epithelial to a mesenchymal phenotype, a process mediated by TGF- $\beta 1$ (33). Chronic peritoneal dialysis induces peritoneal fibrosis, which coincides with the EMT of mesothelial cells and the loss of peritoneal membrane integrity and, consequently, the loss of the ability of the membrane to act as a filter during peritoneal dialysis (2). That mesothelial cells react to both peritoneal dialysis and metastasis with EMT implies that the response of mesothelial cells to cancer cells is not tumor cell specific, but is a generalized reaction of the peritoneum to irritants. Mesothelial cells (45) secrete plasminogen activator inhibitors (PAIs), urokinase (uPA), and VEGF upon local stress (e.g., dialysis and infection). While this has not been directly studied in cancer, it is probable that mesothelial cell secretion of these factors supports metastasis in a manner similar to that described here for their secretion of fibronectin.

Availability of fibronectin to the cancer cells is a critical component for metastasis. This is evidenced by a significant reduction in metastasis or in the ability of cancer cells to invade if fibronectin is knocked out in the peritoneal microenvironment (Figures 4 and 5). Fibronectin has a well-established role in cancer metastasis, pro- moting almost every aspect of tumor progression through activation of mitogenic signaling. In addition, it has known antiapoptotic functions (29), promotes angiogenesis (21), and promotes the recruitment of regulatory $\mathrm{T}$ cells (46). In invasive ovarian tumors, fibronectin expression is found in the ECM, the submesothelial basement membrane of omental metastasis (Figure 1), and in ascites (19) and is associated with an adverse clinical prognosis (47). Moreover, the cleavage of fibronectin into small fragments by MMP-2 has been found to enhance the adhesion of OvCa cells to the peritoneal surface (39). Taken together, these data clearly indicate that fibronectin levels and proteolytic cleavage forms promote the aggressiveness of OvCa cells.

The concept presented here, that the fibronectin secreted by mesothelial cells is important for the metastatic process of $\mathrm{OvCa}$ cells, should have considerable relevance when selecting clinically useful targeted therapies. We found that functionally blocking both $\alpha_{5}$ integrin and $\beta_{1}$ integrin function inhibited the initial metastasis of OvCa cells and reduced the initiation and growth of metastatic tumors (Figure 8 and refs. 48, 49). Furthermore, ATN-161, a short fibronectin peptide binding to $\alpha_{5} \beta_{1}$ and $\alpha_{v} \beta_{3}$ integrins, blocked the initial metastasis of OvCa cells and inhibited the growth of established tumors (Supplemental Figure 8). ATN161 was previously tested in preclinical models of breast and lung cancer (34) and in a clinical phase I study that resulted in patients with solid tumors showing stable disease (36). Our present data suggest that maintenance therapy aimed at blocking the interaction of fibronectin with the fibronectin receptor $\alpha_{5} \beta_{1}$ integrin after the end of adjuvant chemotherapy might delay tumor recurrence and should be considered for further clinical testing. In addition, knocking down fibronectin in mesothelial cells was significantly more efficient in inhibiting OvCa metastasis than solely blocking $\alpha_{5} \beta_{1}$ integrin, which suggests that other integrin and nonintegrin fibronectin receptors contribute to fibronectin-mediated metastasis within the abdominal cavity.

On the basis of our combined data, we propose that the interaction of OvCa cells with the mesothelium is an important functional predeterminant of tumor metastasis and growth (Figure $8 \mathrm{C}$ ). Is it possible, however, to reconcile the observation that OvCa cells displaced mesothelial cells and induced their apoptosis with the finding that mesothelial cells promoted cancer cell metastasis? It may be that the very initial interaction of $\mathrm{OvCa}$ cells with mesothelial cells leads to displacement of some of the mesothelial cells, allowing the OvCa cells to adhere directly to the submesothelial $\operatorname{ECM}(6,27,32)$. The data presented here indicate that after establishing their initial foothold, OvCa cells actively recruit and activate adjacent mesothelial cells. Therefore, the mesothelial cells play a key supporting role in establishing the first metastatic colony on the omentum/peritoneum, acting as cancer-associated mesothelial cells.

\section{Methods}

Further information can be found in Supplemental Methods.

Reagents. Collagen I (rat tail) and fibronectin were purchased from BDBiosciences. Thehuman OvCacelllines, SKOV3ip1andHeyA8, were provided by G.B. Mills (M.D. Anderson Cancer Center, Houston, Texas, USA). The mouse OvCa cell line ID8 was provided by K. Roby (University of Kansas Medical Center, Kansas City, Kansas, USA; ref. 50). 
The cell lines were validated by short tandem repeat DNA fingerprinting using the AmpFlSTR Identifier kit (Applied Biosystems) and compared with known American Type Culture Collection fingerprints, the Cell Line Integrated Molecular Authentication database (CLIMA), and the University of Texas MD Anderson Cancer Center fingerprint database. Negative control, fibronectin, in vivo fibronectin, and vitronectin siRNA were purchased from Ambion Inc.; SMAD3 and TGF- $\beta 1$ siGENOME siRNA were purchased from Dharmacon. TGF- $\beta 1$ was from Gibco. The TGF- $\beta$ RI kinase inhibitor I (catalog no. 616451) (51), TGF- $\beta$ RI kinase inhibitor II (catalog no. 616452) (52), and RHO kinase inhibitor (catalog no. 555552) (53) were from Calbiochem. The RAS signaling activity inhibitor (catalog no. 10010501) (54) was from Cayman Chemical. The RAC-GEF interaction inhibitor (catalog no. 2161) was from Tocris Bioscience (55). The TGF- $\beta$ RII antibody (catalog no. AF-241-NA) was from R\&D Systems (56). The $\beta_{1}$ integrin (clone P5D2) antibody was purchased from Santa Cruz. The fibronectin (clone 568), $\beta_{4}$ integrin (clone ASC-3), $\alpha_{\mathrm{v}} \beta_{3}$ integrin (clone LM609), and $\alpha_{5}$ integrin (clone P1D6) antibodies were purchased from Millipore. The $\beta_{1}$ integrin (OS2966) antibody was provided by OncoSynergy. The mouse $\alpha_{5}$ integrin antibody (clone 339.1) was provided by Facet Biotech Corp. The nonspecific IgG was from Chrome Pure, Jackson ImmunoResearch. Anti-SMAD2/3 (catalog no. 610842) and anti-E-cadherin (catalog no. 610181) antibodies were from BD Transduction Laboratories. Anti-vimentin (clone V9) antibody was purchased from Dako Cytomation. Anti-actin, anti-pSMAD2/3 (clone D6G10), and anti-GAPDH (clone 14C10) antibodies were from Cell Signaling Technology. Ad5CMVCre-eGFP and Ad5CMV-eGFP were purchased from University of Missouri.

TMA and immunohistochemistry. TMA cores $(n=2)$ of omental metastases from 108 patients with serous papillary OvCa were collected (Supplemental Table 3). The patients were selected for the TMAs by a gynecologic pathologist. Clinical and histopathologic information was collected and updated as previously reported (57). TMA slides were deparaffinized and incubated with anti-fibronectin (SigmaAldrich) at 1:400 dilution as previously described $(25,39)$. Slides were stained using the Envision avidin-biotin-free detection system and counterstained with hematoxylin. Quantification and localization of fibronectin protein expression was performed using Aperio ImageScope and Spectrum software. Tumor- and stroma-specific classifiers were developed using a Spectrum analysis program with Genie capabilities (Supplemental Figure 9). The intensity of DAB staining was determined in tumor- or stroma-specific regions of the tumor cores. Differences between tumor and stroma fibronectin expression were evaluated using a Wilcoxon rank test comparing the 2 treatments.

ECM isolation. ECM was isolated from primary human omentum obtained from patients with benign disease and from omental metastases from OvCa patients as previously described (58). Briefly, fresh tissue was cut (1-2 mm thickness), suspended in dispase solution ( $2 \mathrm{mg} / \mathrm{g}$ tissue), and incubated for 2 hours at $4^{\circ} \mathrm{C}$. The tissue sections were rubbed over a cell sieve $(250 \mu \mathrm{m})$, and the remaining matrices were homogenized in high salt buffer $(0.05 \mathrm{M}$ Tris $\mathrm{pH} 7.4,3.4 \mathrm{M} \mathrm{NaCl}$, $4 \mu \mathrm{M} \mathrm{N}$-ethylmaleimide) containing protease inhibitors $(0.001 \mathrm{mg} / \mathrm{ml}$ pepstatin, $0.01 \mathrm{mg} / \mathrm{ml}$ aprotonin, $2 \mathrm{mM}$ orthovanadate, and $1 \mathrm{mM}$ PMSF). The matrices were centrifuged 3 times at 7,000 $g$ for $15 \mathrm{~min}$ utes, and the supernatant was discarded. The pellet was incubated in $2 \mathrm{M}$ urea buffer $(0.15 \mathrm{M} \mathrm{NaCl}, 0.05 \mathrm{M}$ Tris $\mathrm{pH} 7.4)$ at $1 \mathrm{ml}$ buffer $/ \mathrm{g}$ tissue rotating for 48 hours at $4^{\circ} \mathrm{C}$. The solubilized ECMs were centrifuged at
14,000 $g$ for 20 minutes, and supernatant was collected. The concentration and sizes of ECMs isolated were analyzed using BCA protein concentration assay and SDS-PAGE. The protocol was adapted from a method previously described by Abberton et al. (59).

Additionally, the ECM secreted by mesothelial cells was analyzed. Briefly, cells were washed 3 times with PBS and floated off of plate in $0.2 \mathrm{~N} \mathrm{NH}_{4} \mathrm{OH}$. The remaining ECMs were washed 3 times with PBS, collected, and analyzed using SDS-PAGE. The protocol was adapted from a previously described method (60).

qRT-PCR. After coculture of GFP-labeled OvCa cells with full human omentum, the $3 \mathrm{D}$ omental culture, or primary human mesothelial cells, cells were sorted by FACS in PBS (39). This procedure separated labeled OvCa cells from mesothelial cells and/or fibroblasts after their coculture. TRIzOL reagent was used to isolate RNA according to the manufacturer's instructions (Invitrogen). cDNA was synthesized using the Applied Biosystems cDNA archive kit. After reverse transcription, real-time PCR was performed using a Prism7500 TaqMan PCR detector (Applied Biosystems) with predesigned and validated TaqMan probes for FN1, CDH1 (encoding E-cadherin), and $V I M$ (encoding vimentin) in conjunction with GAPDH for normalization (Applied Biosystems) (58). The reactions were run in triplicate. Relative levels of mRNA expression were calculated using the $2^{-\Delta \Delta \mathrm{Ct}}$ method (61). Differences between treatments were evaluated using unpaired, 2-tailed Student's $t$ test.

Immunoblot analysis. For analysis of cellular fibronectin, tissue or cells after FACS were lysed, and equal quantities of protein were added to each blot. For analysis of secreted ECM, equal volumes of ECM lysate were added to each blot. For analysis of tumor ECM, equal quantities of protein were added to each blot. Proteins were resolved by SDS-PAGE and transferred to nitrocellulose, and immunoblot analysis was performed (58). The following antibodies were applied overnight at $4^{\circ} \mathrm{C}$ : anti-fibronectin (1:2,000 dilution); anti-phospho-SMAD2/3 (1:1,000), anti-E-cadherin (1:2,000), anti-vimentin (1:2,000), antiGAPDH (1:2,000), mouse anti-actin (1:50,000). Blots were than incubated with secondary horseradish peroxidase-conjugated $\operatorname{IgG}$ and visualized with enhanced chemiluminescence reagents.

Ex vivo full human omentum culture. For omental cultures, a fresh piece of full human omentum was cut into 8-mm pieces (equivalent weights) (14). All omental pieces were placed in a 24 -well dish (1 piece/well). For the adhesion assay, full human omentum pieces were transfected with in vivo fibronectin or control siRNA 24 hours prior to coculture with $1 \times 10^{6}$ fluorescently labeled SKOV3ip 1 cells. After incubation at $37^{\circ} \mathrm{C}$ for 4 hours, omental pieces were washed 3 times in PBS, digested in $5 \% \mathrm{NP}-40$ for 30 minutes at $37^{\circ} \mathrm{C}$, and scraped with a metal spatula. All cells removed during digestion were placed in a 24-well plate, and the total fluorescent intensity per well was quantified. Adhesion assays were run in quadruplicate.

For coculture assays, full human omentum pieces were cultured with $1 \times 10^{6}$ SKOV3ip1 cells. After incubation at $37^{\circ} \mathrm{C}$ for 48 hours, omental pieces were digested in $0.2 \%$ trypsin-EDTA for 5 minutes at $37^{\circ} \mathrm{C}$ and scraped with a metal spatula. The trypsin was neutralized, and collected cells were pelleted. For analysis of fibronectin expression and production, GFP-labeled SKOV3ip1 cells and omental surface cells were sorted by FACS.

Primary omental cells and $3 D$ omental culture. Primary human mesothelial cells and fibroblasts were isolated from the omentum, and purification was verified by vimentin, keratin 8 , and prolyl-hydroxylase 
immunohistochemistry $(25,39)$. The 3D omental culture system was assembled by plating 4,000 human primary fibroblasts per well plus $0.5 \mu$ collagen type I. 4 hours later, human primary mesothelial cells were added at a density of 20,000 cells in $0.33 \mathrm{~cm}^{2}$.

Adhesion assay. Omental ECM $(0.5 \mu \mathrm{g})$, tumor ECM $(0.5 \mu \mathrm{g})$, or 20,000 primary human mesothelial cells were used to coat a 96 -well dish $\left(0.33 \mathrm{~cm}^{2}\right)$, and a 1-hour adhesion assay was performed with 40,000 fluorescently labeled OvCa cells (39). Cells were treated with mouse IgG, $\beta_{1}$ integrin, $\alpha_{5}$ integrin, $\alpha_{\mathrm{v}} \beta_{3}$ integrin, or $\beta_{4}$ integrin antibodies $(20 \mu \mathrm{g} / \mathrm{ml})$ at the time of cell plating. Differences between treatments were evaluated using unpaired, 2-tailed Student's $t$ test.

Invasion assay. 50,000 fluorescently labeled OvCa cells were plated on each well of a 24-well transwell plate $\left(0.33 \mathrm{~cm}^{2}\right)$ that was precoated with $20 \mu \mathrm{g}$ omental ECM, precoated with $20 \mu \mathrm{g}$ tumor ECM, or contained $20 \mu \mathrm{g}$ collagen type I with 20,000 primary human mesothelial cells. Cells were treated with mouse $\operatorname{IgG}, \beta_{1}$ integrin, $\alpha_{5}$ integrin, $\alpha_{v} \beta_{3}$ integrin, or $\beta_{4}$ integrin antibodies $(20 \mu \mathrm{g} / \mathrm{ml})$ at the time of cell plating. The invasion assays were performed as previously described (39). Differences between treatments were evaluated using unpaired, 2-tailed Student's $t$ test.

IF. GFP-labeled SKOV3ip1 cells were added to a culture of primary human mesothelial cells or fibronectin-coated $(100 \mu \mathrm{g})$ glass coverslips for 48 hours (25). The cells were fixed with $4 \%$ paraformaldehyde in PBS for 30 minutes, and IF was performed. Primary antibodies against fibronectin (1:400 dilution) and secondary antibodies (goat anti-rabbit Alexa Fluor 645, 1:200 dilution; Invitrogen) were used. Intracellular fibronectin was analyzed using Triton-X detergent in buffers $(0.1 \%)$, while extracellular fibronectin was analyzed using buffers lacking detergents. The Triton-X detergent permeabilizes the cell membrane, allowing for intracellular fibronectin detection while washing away extracellular fibronectin. Nuclear counterstain was performed with Hoechst. Imaging was performed using a Zeiss LSM510 confocal microscope.

DOC solubility assay. The assay was based on the insolubility of fibronectin matrix in 2\% DOC detergent (62). Cell pellets were lysed in DOC lysis buffer (2\% sodium DOC, 20 mM Tris-HCl pH 8.8, 2 mM PMSF, 2 mM EDTA, 2 mM iodoacetic acid, 2 mM N-ethylmaleimide). Lysates were centrifuged to separate DOC-insoluble matrix from DOC-soluble material containing cell-associated and intracellular fibronectin. The DOC-insoluble fibronectin was solubilized in a buffer containing 1\% SDS (20 mM Tris- $\mathrm{HCl}$ pH 8.8, 2 mM PMSF). The DOC-soluble and -insoluble fractions were resolved by SDS-PAGE, transferred to nitrocellulose, and analyzed by immunoblotting.

Inhibition experiments. Human OvCa cell lines SKOV3ip1 and HeyA8 were added to a confluent culture of primary human mesothelial cells and treated with RAC inhibitor $(5 \mu \mathrm{m})$, RAS inhibitor $(25 \mu \mathrm{m})$, RHO inhibitor $(10 \mu \mathrm{m})$, TGF- $\beta$ RI inhibitors $(10 \mu \mathrm{m})$, or anti-TGF$\beta$ RII $(8 \mu \mathrm{g} / \mathrm{ml})$ neutralizing antibody. Fibronectin production and secretion was analyzed 48 and 72 hours later, respectively. Fibronectin, SMAD3, TGF- $\beta$ RII, and control siRNA constructs were transfected into $2 \times 10^{6}$ primary human mesothelial cells 18 hours prior to coculture with SKOV3ip1 cells. SKOV3ip1 cells were transfected with TGF- $\beta 1$ or control siRNA constructs using Lipofectamine Transfection Reagent (Invitrogen). The cells recovered in full growth media (10\% fetal bovine serum) for 18 hours, and serum-free media was added. SKOV3ip1 cell CM was collected after 48 hours and concentrated (10x) using a spin column with 3- $\mu \mathrm{m}$ filter (Millipore).
RAC activity measurement. Immunoprecipitation-immunoblot was performed as described by the manufacturer (Cell Biolabs Inc.). Briefly, $1 \times 10^{7}$ SKOV3ip1 cells were cultured alone or added to a culture of $4 \times 10^{6}$ primary human mesothelial cells for 24 hours. Cells were washed with PBS, lysed, scraped, collected, and pelleted. The supernatant was incubated with $0.05 \mathrm{M}$ EDTA for 30 minutes at $30^{\circ} \mathrm{C}$. GTP $\gamma$ S or GDP were added to duplicate samples for positive and negative controls, and loading was quenched with $0.2 \mathrm{M} \mathrm{MgCl}_{2}$ treatment. RAC1 pulldown assay was performed. Cell lysates were incubated with PAK PBD agarose beads and incubated at $4^{\circ} \mathrm{C}$ for 1 hour. The beads were pelleted, washed, and boiled in $2 \times$ reducing SDS-PAGE sample buffer. The supernatant was resolved by SDS-PAGE and transferred to nitrocellulose, and immunoblot was performed. Anti-RAC1 antibody (1:1,000 dilution) was applied overnight at $4^{\circ} \mathrm{C}$. Blots were then incubated with secondary horseradish peroxidase-conjugated IgG and visualized with enhanced chemiluminescence reagents.

Transfections. Primary human mesothelial cells were transiently transfected with the full-length FN1 promoter (-1,200 bp) (28) using Lipofectamine Transfection Reagent (Invitrogen) (39). At 18 hours after transfection, SKOV3ip1 cells $\left(1.5 \times 10^{6} /\right.$ well $)$ were trypsinized and added on the transfected mesothelial cells. After 12 hours, cells were detached and lysed, and luciferase was analyzed (63).

In vivo mouse OvCa coculture. Athymic nude mice received i.p. injection of $4 \times 10^{6}$ HeyA8 cells (39). 48 hours after injection, the mouse omentum was removed, digested (0.2\% trypsin-EDTA) for 5 minutes at $37^{\circ} \mathrm{C}$, and scraped with a metal spatula. The trypsin was neutralized, collected cells were pelleted, and fibronectin production was analyzed.

Fn1 $1^{f / f}$ mouse experiments. Fn1 $1^{f / f}$ mice - generated by R. Fässler (Max-Planck Institute of Biochemsitry, Martinsried, Germany) and provided by S. Dallas (University of Missouri, Kansas City, Missouri, USA) - have been previously described $(29,64)$. The mice were bred in house and regularly genotyped (29). $F n 1^{f / f l}$ mice have no reported or observed abnormalities, are fertile, and have a normal lifespan.

In order to generate mice with tissue-specific deletion of Fn1, Cre must be expressed in a tissue-specific and/or inducible manner (29). Therefore, $F n 1^{A / / A}$ mice received i.p. injections of Ad-Cre (Ad5CMVCre) to delete fibronectin in the lining of the peritoneal cavity, or i.p. injections of Ad-Cont (Ad5CMV), 96 hours prior to cancer cell injection. $4 \times 10^{6}$ GFP-labeled ID8 mouse OvCa cells (65) were injected; 72 hours later, the mouse omentum was removed and imaged by fluorescent microscopy. ID8 and omental surface cells were digested (0.1\% NP-40) off of the omentum, and total fluorescence of bound cells was measured using a fluorescent plate reader (BioRad). Adenovirus infection (GFPlabeled cells) was confirmed in peritoneal surface cells, and allele-specific PCR validated the recombination of floxed $F n 1$ in peritoneal surface cells 48 hours after i.p. adenovirus injection. To test the effect of an $\alpha_{5}$ integrin blocking antibody in addition to fibronectin knockdown, $4 \times 10^{6}$ GFP-labeled ID8 cells were preincubated for 15 minutes with $20 \mu \mathrm{g} / \mathrm{ml}$ $\alpha_{5}$ integrin antibody (clone 339.1) or mouse IgG before i.p. injection.

Peritoneal surface cells from $F n 1^{f / f t}$ mice were isolated, cultured, and infected with Ad-Cre or Ad-Cont. Adhesion, invasion, and proliferation of ID8 cells were examined on the adenovirus-infected peritoneal surface cells, as described above. The adenovirus infection was efficient (>92\%; infection at MOI 50), as observed with eGFP expression, and did not reduce viability $(\mathrm{MOI}<100)$. Recombination of floxed Fn1 was verified by allele-specific PCR (see below). 
Treatment studies. The effect of $\alpha_{5}$ integrin (66) or $\beta_{1}$ integrin (38) antibody on OvCa cell adhesion, invasion, and proliferation in vitro was investigated. Mouse IgG (control) or $\alpha_{5}$ integrin or $\beta_{1}$ integrin antibody $(10 \mu \mathrm{g} / \mathrm{ml})$ was added to fluorescently labeled SKOV3ip1 and HeyA8 OvCa cells 15 minutes prior to performing assay and adhesion (2 hours), invasion (24 hours), or proliferation (96 hours) assays on the preplated 3D omental culture (25).

For the in vivo study, $2.5 \times 10^{4}$ HeyA8 cells were mixed with mouse IgG (control) or $\alpha_{5}$ integrin or $\beta_{1}$ integrin antibody $(10 \mu \mathrm{g} / \mathrm{ml})$, and $10 \mu \mathrm{l}$ was injected into the ovaries of athymic nude mice. At 2 and 4 days after injection, mouse IgG or $\alpha_{5}$ integrin or $\beta_{1}$ integrin antibody in PBS $(10 \mathrm{mg} / \mathrm{kg} / \mathrm{d})$ was administered i.p. At 28 days after injection, mice were sacrificed (10 per group). The number of tumor colonies was counted, and the tumor was weighed (57).

Statistics. Adhesion $(n=5)$ and invasion $(n=3)$ assays were performed and at least 3 independent experiments conducted. Mean and SEM are reported. Significance of differences was determined by 2-tailed independent (unpaired) $t$ test; a $P$ value less than 0.05 was considered significant.

Study approval. Specimens of human omentum were obtained from patients undergoing surgery for benign conditions, and tumor samples from OvCa patients undergoing surgery, at the University of Chicago. All human specimens were obtained in accordance with a protocol approved by the University of Chicago IRB, and subjects provided informed consent. All animal procedures were approved by the University of Chicago IACUC and were in accordance with the University of Chicago's policies on the care, welfare, and treatment of laboratory animals.

\section{Acknowledgments}

We thank A.F. Haney (Department of Obstetrics and Gynecology, University of Chicago) for collecting omental biopsies, G. Isenberg (University of Chicago) for editing the manuscript, A. Mitra for helpful discussions, R. Fassler and S. Dallas for the $F n 1^{f / A}$ mice, S. Carbonell and OncoSynergy for providing the $\beta_{1}$ integrin antibody, K. Roby for providing the ID8 cell line, G.B. Mills for providing the HeyA8 and SKOV3ip1 cell lines, and the human tissue resource center (Terri Li and Lei-Ann Arceneaux) and flow cytometry (Ryan Duggan) core facilities at the University of Chicago for expert technical support. This work was supported by NCI grant R01 CA111882 and by an Ovarian Cancer Research Fund Program Project Development Grant to E. Lengyel. Finally, we appreciate all the patients, resident physicians, and attending physicians in the Department of Obstetrics and Gynecology at the University of Chicago for their commitment in collecting tissue.

Address correspondence to: Ernst Lengyel, University of Chicago, Department of Obstetrics and Gynecology/Section of Gynecologic Oncology, 5841 S. Maryland Avenue, Chicago, Illinois 60637, USA. Phone: 773.834.0563; E-mail: elengyel@uchicago.edu.
1. Lengyel E. Ovarian cancer development and metastasis. Am J Pathol. 2010;177(3):1053-1064.

2. Mutsaers SE. The mesothelial cell. Int J Biochem Cell Biol. 2004;36(1):9-16.

3. Zhang XY, Pettengell R, Nasiri N, Kalia V, Dalgleish AG, Barton DP. Characteristics and growth patterns of human peritoneal mesothelial cells: Comparison between advanced epithelial ovarian cancer and non-ovarian cancer sources. J Soc Gynecol Investig. 1999;6(6):333-340.

4. Niedbala MJ, Crickard K, Bernacki R. Interactions of human ovarian tumor cells with human mesothelial cells grown on extracellular matrix. Exp Cell Res. 1985;160(2):499-513.

5. Iwanicki M, et al. Ovarian cancer spheroids use myosin-generated force to clear the mesothelium. Cancer Discov. 2011;1(7):144-157.

6. Davidowitz RA, et al. Mesenchymal gene program-expressing ovarian cancer spheroids exhibit enhanced mesothelial clearance. J Clin Invest. 2014;124(6):2611-2625.

7. Heath RM, Jayne DG, O'Leary R, Morrison EE, Guillou PJ. Tumor-induced apoptosis in human mesothelial cells: a mechanism of peritoneal invasion by Fas Ligand/Fas interaction. BrJCancer. 2004;90(7):1437-1442.

8. Rieppi M, et al. Mesothelial cells induce the motility of human ovarian carcinoma cells. Int J Cancer. 1999;80(2):303-307.

9. Ksiazek K, Mikula-Pietrasik J, Korybalska K, Dworacki G, Jorres A, Witowski J. Senescent peritoneal mesothelial cells promote ovarian cancer cell adhesion: The role of oxidative stress-induced fibronectin. Am J Pathol. 2009;174(4):1230-1240.

10. Hanahan D, Coussens LM. Accessories to the crime: Functions of cell recruited to the tumor microenvironment. Cancer Cell. 2012; 21(3):309-322.

11. Mueller M, Fusenig N. Friends or foes - bipolar effects of the tumour stroma in cancer. Nat Rev Cancer. 2004;4(11):839-849.

12. Granot D, Addadi Y, Kalchenko V, Harmelin A, Kunz-Schughart LA, Neeman M. In vivo imaging of the systemic recruitment of fibroblasts to the angiogenic rim of ovarian carcinoma tumors. Cancer Res. 2007;67(19):9180-9189.

13. Schauer IG, Sood AK, Mok S, Liu J. Cancer-associated fibroblasts and their putative role in potentiating the initiation and development of epithelial ovarian cancer. Neoplasia. 2011;13(5):393-405.

14. Nieman KM, et al. Adipocytes promote ovarian cancer metastasis and provide energy for rapid tumor growth. Nat Med. 2011;17(11):1498-1503.

15. Nieman KM, Romero IL, Van Houten B, Lengyel E. Adipocyte tissue and adipocytes support tumorigenesis and metastasis. Biochim Biophys Acta. 2013;1831(10):1533-1541.

16. Burleson KM, Casey RC, Skubitz KM, Pambuccian SE, Oegema TR, Skubitz AP. Ovarian carcinoma ascites spheroids adhere to extracellular matrix components and mesothelial cell monolayers. Gynecol Oncol. 2004;93(1):170-181.

17. Cannistra SA, Ottensmeier C, Niloff J, Orta B, DiCarlo J. Expression and function of $\beta 1$ and $\alpha v \beta 3$ integrins in ovarian cancer. Gynecol Oncol. 1995;58(2):216-225.

18. Bignotti E, et al. Gene expression profile of ovarian serous papillary carcinomas: identification of metastasis-associated genes. Am JObstet Gynecol. 2007;196(3):245.e1-11.

19. Wilhelm O, Hafter R, Coppenrath E, Pflanz M,
Schmitt M, Graeff H. Fibrin-fibronectin compounds in human ovarian tumor ascites and their possible relation to the tumor stroma. Cancer Res. 1988;48(12):3507-3514.

20. Mould AP, et al. Defining the topology of integrin $\alpha 5 \beta 1$-fibronectin interactions using inhibitory anti- $\alpha 5$ and anti- $\beta 1$ monoclonal antibodies. J Biol Chem. 1997;272(28):17283-17292.

21. Desgrosellier JS, Cheresh D. Integrins in cancer: Biological implications and therapeutic opportunities. Nat Rev Cancer. 2010;10(1):9-22.

22. Schwarzbauer JE, Sechler JL. Fibronectin fibrillogenesis: a paradign for fibronectin matrix assembly. Curr Opin Cell Biol.1999;11(5):622-627.

23. Mitra AK, Sawada K, Tiwari P, Mui K, Gwin K, Lengyel E. Ligand-independent activation of $c$-Met by fibronectin and $\alpha 5 \beta 1$-integrin regulates ovarian cancer invasion and metastasis. Oncogene. 2011;30(13):1566-1576.

24. Zand L, Feng Q, Roskelley CD, Leung PCK, Auersperg N. Differential effects of cellular fibronectin and plasma fibronectin on ovarian cancer cell adhesion, migration, and invasion. Int J Biochem Cell Biol. 2003;39(3-4):178-182.

25. Kenny HA, Krausz T, Yamada SD, Lengyel E. Use of a novel 3D culture model to elucidate the role of mesothelial cells, fibroblasts and extra-cellular matrices on adhesion and invasion of ovarian cancer cells. Int J Cancer. 2007;121(7):1463-1472.

26. Daya D, McCaughy WT. Pathology of the peritoneum: A review of selected topics. Semin Diagn Pathol. 1991;8(4):277-289.

27. Kenny HA, Nieman KM, Mitra AK, Lengyel E. The first line of intra-abdominal metastatic attack: Breaching the mesothelial cell layer. Cancer Discov. 2011;1(2):100-102. 
28. Michaelson JE, Ritzenthaler J, Roman J. Regulation of serum-induced fibronectin expression by protein kinases, cytoskeletal integrity, and CREB. Am J Physiol Lung Cell Mol Physiol. 2002;282(2):L291-L301.

29. Sakai T, et al. Plasma fibronectin supports neuronal survival and reduces brain injury following transient focal cerebral ischemia but is not essential for skin-wound healing and hemostasis. Nat Med. 2001;7(3):324-330.

30. Yang J, et al. Twist, a master regulator of morphogenesis, plays an essential role in tumor metastasis. Cell. 2004;117(7):927-939.

31. Tothill RW, et al. Novel molecular subtypes of serous and endometrioid ovarian cancer linked to clinical outcome. Clin Cancer Res. 2008;14(16):198-208.

32. Sandoval P, et al. Carcinoma-associated fibroblasts derive from mesothelial cells via mesothelial to mesenchymal transition in peritoneal metastasis. J Pathol. 2013;231(4):517-531.

33. Yáñez-Mó $M$, et al. Peritoneal dialysis and epithelial-to-mesenchymal transition of mesothelial cells. N Engl J Med. 2003;348(5):403-413.

34 . Yamamura $S$, et al. The activated transforming growth factor- $\beta$ signaling pathway in peritoneal metastases is a potential therapeutic target in ovarian cancer. Int J Cancer. 2012;130(1):20-28.

35. Yamamoto $\mathrm{H}$, et al. Seperate roles for $\mathrm{H}-\mathrm{Ras}$ and Rac in signaling by transforming growth factor (TGF)- 3 . Eur J Biochem. 1999;264(1):110-119.

36. Tse JC, Kalluri R. Mechanisms of metastasis: epithelial-to-mesenchymal transition and contribution of tumor microenvironment. J Cell Biochem. 2007;101(4):816-829.

37. Adib TR, Henderson S, Perrett C, Bourmpoulia D, Lederman J, Boshoff C. Predicting biomarkers for ovarian cancer using gene-expression microarrays. Br J Cancer. 2004;90(3):686-692.

38. Carbonell WS, DeLay M, Jahangiri A, Park CC, Aghi MK. beta1 integrin targeting potentiates antiangiogenic therapy and inhibits the growth of bevacizumab-resistant glioblastoma. Cancer Res. 2013;73(10):3145-3154.

39. Kenny HA, Kaur S, Coussens LM, Lengyel E. The initial steps of ovarian cancer cell metastasis are mediated by MMP-2 cleavage of vitronectin and fibronectin. JClin Invest. 2008;118(4):1367-1379.

40. Olumi AF, Grossfeld GD, Hayward SW, Carroll PR, Tlsty TD, Cunha GR. Carcinoma-associated fibroblasts direct tumor progression of intiated human prostatic epithelium. Cancer Res. 1999;59(19):5002-5011.
41. Dirat B, et al. Cancer-associated adipocytes exhibit an activated phenotype and contribute to breast cancer invasion. Cancer Res. 2011;71(7):2455-2465.

42. Aroeira L, et al. Epithelial to mesenchymal transition and peritoneal membrane failure in peritoneal dialysis patients: pathologic significance and potential therapeutic interventions. JAm Soc Nephrol. 2007;18(7):2004-2013.

43. Zavadil J, et al. Genetic programs of epithelial cell plasticity directed by transforming growth factor- $\beta$. Proc Natl Acad Sci U S A. 2001;98(12):6686-6691.

44. Han G, Lu SL, He W, Corless CL, Kulesz-Martin $\mathrm{M}$, Wang XJ. Distinct mechanisms of TGF- $\beta 1$ -mediated epithelial-to-mesenchymal transition and metastasis during skin carcinogenesis. JClin Invest. 2005;115(7):1714-1723.

45. Holmdahl L. The role of fibrinolysis in adhesion formation. Eur J Surg. 1997;(577):24-31.

46. Sengupta S, Nandi S, Hindi ES, Wainwright DA, Han Y, Lesniak MS. Short hairpin RNAmediated fibronectin knowkdown delays tumor growth in a mouse glioma model. Neoplasia. 2010;12(10):837-847.

47. Franke EE, von Georgi R, Zygmunt M, Munstedt K. Association between fibronectin expression and prognosis in ovarian carcinoma. Anticancer Res. 2003;25(118):1331-1339.

48. Sawada K, Ohyagi-Hara C, Kimura T, Morishige Ki. Integrin inhibitors as a therapeutic agent for ovarian cancer. JOncol. 2012;2012:915140.

49. Jahangiri A, Aghi MK, Carbonell WS. beta1 integrin: Critical path to antiangiogenic therapy resistance and beyond. Cancer Res. 2014;74(1):3-7.

50. Roby KF, et al. Development of a syngeneic mouse model for events related to ovarian cancer. Carcinogenesis. 2000;21(4):585-591.

51. Sawyer JS, et al. Synthesis and activity of new aryl- and heteroaryl-substituted pyrazole inhibitors of the transforming growth factor- $\beta$ type I receptor kinase domain. JMed Chem. 2003;46(19):3953-3956.

52. Gellibert F, et al. Identification of 1,5-naphthyridine derivatives as a novel series of potent and selective TGF- $\beta$ type I receptor inhibitors. JMed Chem. 2004;47(18):4494-4506.

53. Ye X, Wang Y, Rattner A, Nathans J. Genetic mosaic analysis reveals a major role for frizzled 4 and frizzled 8 in controlling ureteric growth in the developing kidney. Development. 2011;138(6):1161-1172.

54. George J, et al. Functional inhibition of Ras by
S-trans, trans-farnesyl thiosalicylic acid attenuates atherosclerosis in apolipoprotein $\mathrm{E}$ knockout mice. Circulation. 2002;105(20):2416-2422.

55. Gao Y, Dickerson JB, Guo F, Zheng J, Zheng Y. Rational design and characterization of a Rac GTPase-specific small molecule inhibitor. Proc Natl Acad Sci U S A. 2004;101(20):7618-7623.

56. Tsang ML, et al. Characterization of recombinant soluble human transforming growth factor- $\beta$ receptor type II (rhTGF- $\beta$ sRII). Cytokine. 1995;7(5):389-397.

57. Sawada K, et al. C-Met overexpression is a prognostic factor in ovarian cancer and an effective target for inhibition of peritoneal dissemination and invasion. Cancer Res. 2007;67(4):1670-1680.

58. Kenny HA, et al. Targeting the urokinase plasminogen activator receptor inhibits ovarian cancer metastasis. Clin Cancer Res. 2011; 17(3):459-471.

59. Abberton KM, et al. Myogel, a novel, basement membrane-rich, extracellular matrix derived from skeletal muscle, is highly adipogenic in vivo and in vitro. Cells Tissue Organs. 2008;188(4):347-358.

60. Gospodarowicz D, Cheng J, Lui GM, Baird A, Böhlent P. Isolatin of brain fibroblast growth factor by heparin-sepharose affinity chromatography: I dentitiy with pituitary fibroblast growth factor. Proc Natl Acad Sci US A. 1984;81(22):6963-6967.

61. Pfaffl M. A new mathematical model for relative quantification in real-time RT-PCR. Nucleic Acids Res. 2001;29(9):2002-2007.

62. McKeown-Longo PJ, Mosher D. Binding of plasma fibronectin to cell layers of human skin fibroblasts. JCell Biol. 1983;97(2):466-472.

63. Hapke S, et al. $\beta 3$ A-integrin downregulates the urokinase-type plasminogen activator receptor (u-PAR) through a PEA3/ets transcriptional silencing element in the u-PAR promoter. Mol Cell Biol. 2001;21(6):2118-2132.

64. Brunner M, et al. Osteoblast mineralization requires $\beta 1$ integrin/ ICAP-1-dependent fibronectin deposition. J Cell Biol. 2011;194(2):307-322.

65. Indraccolo S, et al. Gene transfer in ovarian cancer cells: A comparison between retroviral and lentiviral vectors. Cancer Res. 2002;62(21):6099-6107.

66. Bhaskar V, et al. A function blocking anti-mouse integrin $\alpha 5 \beta 1$ antibody inhibits angiogenesis and impedes tumor growth in vivo. J Transl Med. 2007;5(61):1-11. 